\title{
Voix narrative hypertextuelle et cohérence sémantique dans L'Affaire Le Moine Étude sur le pastiche de Sain-Simon par Proust
}

\author{
José SALAS Quirós \\ Escuela de Lenguas Modernas \\ Universidad de Costa Rica
}

\begin{abstract}
Résumé
Cet article suppose une étude particulière du pastiche du duc de Saint-Simon par Marcel Proust, tout en utilisant deux " grandes notions " (proposées et définies ici même) qui vont traverser le texte et sa composition, à savoir : voix narrative hypertextuelle (d'après les termes structuralistes) et cohérence sémantique (pour ériger une théorie de genèse et structure du texte). C'est à dire qu'il n'y a que deux grandes questions à la base de cette analyse : comment a fonctionné la création de ce pastiche et quels sont les enjeux respectifs de sa narration? puisque nous trouvons que la narration est ce qu'il y a de plus spécial dans l'œuvre et qu'il faut étudier tantôt son origine, tantôt son résultat final. Les réponses s'y déploient selon une méthode d'analyse approfondie des parties du texte et du texte tout ensemble comme unité et tout en suivant une division stricte fondée sur le caractère spécifique de chacune de deux questions exposées.
\end{abstract}

Mots clés: narratologie, hypertextualité, cohérence sémantique, pastiche, Marcel Proust

\begin{abstract}
Resumen
El presente artículo supone un estudio particular del pastiche del duque SaintSimon por Marcel Proust, con la ayuda de dos "grandes nociones" (propuestas y definidas aquí mismo) que cruzan todo el texto y su composición, a saber: voz narrativa hipertextual (según la terminología estructuralista) y coherencia semántica (con el fin de establecer una teoría de creación y estructura del texto). Es decir que nos enfrentamos a dos grandes preguntas en este análisis: ¿cómo funcionó la creación de este pastiche y cuáles son las características respectivas a su narración? dado que consideramos que la narración es lo más especial y remarcable en esta obra y que debe estudiarse tanto su origen como su resultado final. Las respuestas se despliegan según un método de profundo análisis de las partes del texto y del texto como unidad, siempre respetando la estricta división basada en el carácter especifico de cada una de las preguntas expuestas.
\end{abstract}

Palabras claves: narratología, hipertextualidad, coherencia semántica, pastiche, Marcel Proust 
Mais d'une part les vérités de l'intelligence, si elles sont moins précieuses que ces secrets du sentiment dont je parlais tout à l'heure, ont aussi leur intérêt $t^{1}$.

\section{A. Introduction}

L a présente étude porte sur quelques faits littéraires (dont notamment deux auxquels on reviendra à son moment) qui se dégagent de la lecture d'un texte particulier et succinct de Marcel Proust : le pastiche du duc de Saint-Simon, l'un des composants majeurs de l'ouvrage : Pastiches et Mélanges publié en 1919². D'où le recours que l'on fait ici de la tout particulière graphie : "Le Moine ", forme archaïsante (effet imitatif) ${ }^{3}$, pour ainsi dire, du nom "Lemoine ". Ce n'est pas donc absolument notre volonté de faire rebaptiser le pastiche ; nommé plus normalement (lorsqu'il est pris singulièrement et d'après le titre que Proust lui-même lui a conféré) : L'Affaire Lemoine, dans les Mémoires de Saint-Simon, mais il s'agit de repérer le plus vite possible la particularité de cet écrit par rapport aux autres pastiches qui l'accompagnent dans le recueil cité plus haut et de poser, peut-être surtout, l'accent sur un fait du texte qui deviendra un axe essentiel pour la suite de cette étude.

En effet, l'une des caractéristiques les plus remarquables et les plus évidentes du pastiche en question, c'est la présence des manchettes (ou sous-titres, où l'on repère la graphie : Le Moine), trait distinctif du mémorialiste, y convoquées pour des propos imitatifs. Certes, on ne veut pas encore rentrer dans la description spécifique du pastiche, qui aura son lieu plus tard, mais on a la volonté manifeste de relever l'importance de cette partie "limite » de la littérature, nommée ailleurs : paratextualité ${ }^{4}$ Ainsi osons-nous appeler le texte L'Affaire Le Moine, par le biais d'une forme plus interne mais toujours liminaire de sa constitution, et d'élever, en amont, cette particularité au rang de reconnaissance extérieure.

Revenant donc du côté de la cohérence interne du texte, il est deux phénomènes, annoncés plus haut, qui recevront le plus d'attention tout au long de cet exposé, à savoir : les enjeux esthétiques et éthiques de la narration, principalement de la voix narrative, dans ce texte imitatif et la place qui revient à la cohérence (au sens d'unité) sémantique, pour établir une éventuelle étude de type général (rhétorique ?) sur cette œuvre. Soit alors, d'une part un essai de proposer une perspective (que non une théorie !) quelque peu nouvelle tant elle est nourrie par deux "systèmes " antérieurement constitués (que l'on signalera quand il en sera question) et dont le mérite, s'il y en a, serait d'agencer, à bon escient, une convergence pertinente de ces deux systèmes, qui puisse révéler un aspect jusqu'ici presque inconsidéré de ce pastiche. De l'autre part, l'intention de justifier le fait d'ériger la cohérence sémantique au centre d'une analyse portant à son tour sur divers aspects du texte, tant l'unité sémantique (caractéristique proustienne par excellence ${ }^{5}$ ) n'y fait pas l'exception. 
Aussi, nous faudra-t-il passer par différents niveaux et perspectives de la littérarité (au sens de Jakobson ${ }^{6}$ ) du pastiche L'Affaire Le Moine, dont notamment une qui serait susceptible de se poser comme point de convergence des deux grands axes de ce travail. C'est le cas de l'ancien ethos rhétorique, vers lequel vont se diriger dans une certaine mesure les deux buts visés du travail, proposés préalablement. Or, cette "tendance " de la logique argumentative ne rend point invalide le traitement que l'on a décidé de conférer aux problèmes soulevés (qu'il nous manque encore de bien cerner, on en est bien conscient) puisque il s'agit là d'une catégorisation du texte qui répond, comme on le verra, à sa nature précise, et qui pourrait, bien entendu, rentrer dans les considérations des autres niveaux, qu'ils fassent partie du cadre perspectif rhétorique ou non.

Selon la rhétorique classique, on le sait tous, il y existe trois types de discours (ceci principalement à partir de l'intention et de la fonction qu'ils avaient auprès du public récepteur) : délibératif, judiciaire et le plus remarquable à notre égard : épidéictique ${ }^{7}$. C'est particulièrement dans cette direction que certains chercheurs ${ }^{8}$ ont voulu classer ce pastiche ; quant à nous, on parlera à sa place des enjeux éthiques qui dérivent de cette réalité. Cette nature éthique de la discursivité rhétorique du texte, pour ainsi la nommer, ayant été dûment amorcée et cernée ailleurs, on s'en servira ; ne pouvant ignorer la grande valeur d'une contribution pareille. Pourtant le recours que l'on en puisse avoir ici, ne fait que marquer un point coïncidant dans les cheminements que l'on a entrepris. Une fois encore : il s'agit d'une caractéristique qui est susceptible d'affecter dans une mesure ou dans une autre, les possibles regards sur ce texte. Ainsi, dans notre cas, le caractère épidéictique du discours prend la place de convergence à l'intérieur du schéma logique suivi ; tandis que rien n'empêche qu'il puisse occuper celle de divergence notamment, ou d'autres encore, dans un schéma logique qui soit conçu différemment.

Cette explication tient à prévenir toute possible confusion sur ce point. Évidemment l'ensemble du travail pourrait prendre un autre avatar logique dans lequel l'ethos du texte soit pris dans son unité et qu'il ne soit pas partagé par deux autres phénomènes, dont il ne comporte qu'un aspect parmi d'autres. Mais on en a fait autrement, car l'accent est tout simplement placé ailleurs (dans la voix narrative et dans la cohérence sémantique) et que l'on amenuiserait le gros du travail, si l'on choisissait de s'infléchir, à tort, sur une autre ligne de raisonnement dans laquelle l'emphase centrale serait l'ethos littéraire et qui n'apporterait pas pour autant les résultats souhaités. Puisque l'on vient de traiter et la logique et les raisonnements de notre étude, il serait nécessaire que l'on s'y attarde encore quelques instants.

De prime abord, on doit signaler que le développement général qui s'ensuit est fait au rebours du titre accordé pour la recherche. C'est dire que les commentaires portant sur la place et sur la fonctionnalité de la cohérence sémantique y seront traités en premier lieu. Ce qui laisse entendre, et pour cause, que nous allons ensuite traiter les enjeux renfermés dans la circonstance de la voix narrative, attente aucunement trompée ; on n'y envisage pas une structure par'hyponoian, mais une structure qui garde pour sa dernière partie ce qui est considéré 
de plus important. Simple question de choix donc. Par conséquent, nous semblet-il, tantôt le titre, tantôt la structure du texte, privilégient chacun à sa façon le sujet de la voix narrative ; véritable objet innovateur de cette réflexion, puisqu'il reste toujours, jusqu'à notre connaissance, sans être traité sous cette perspective précise.

De cette manière le procédé global de cette enquête consistera à établir une accumulation suffisante des données, faisant état des circonstances requises (définitions, repères historiques, caractéristiques, enfin des jalons) pour partir à la quête des phénomènes amorcés plus haut. Assurément il n'y est pas question de faire un aperçu général de la pratique imitative du pastiche, ni même de la totalité ${ }^{9}$ de Pastiches et Mélanges, mais de borner progressivement la direction de la réflexion en cours, pour pouvoir déverser sur un champ beaucoup plus restreint et plus concentré, où l'on pourra rencontrer, espérons-le, les réponses aux questionnements soulevés par la lecture du pastiche L'Affaire Le Moine.

Or, il est temps justement de soulever les problématiques qui sont à l'origine de cet exercice, nommons-le ainsi par l'instant, et qui y accomplissent le rôle des guides. Pour ce qui est de la cohérence sémantique, on s'est peut-être approché, à notre insu ou tout au plus sans en avoir la moindre intention, à une démarche quelque peu comparative. Deux procédés ayant été identifiés comme étant plausiblement parallèles, et qui réduits à la base peuvent se présenter comme il suit : l'utilisation, déjà remarquée d'ailleurs, des manchettes chez Saint-Simon, et l'hypothèse d'après laquelle ces manchettes pourraient, dans une certaine mesure, déterminer le champ lexical, soit sémantique, de la partie développée qui lui correspond. Du côté de chez Proust (faisons le calembour !), on a repéré un phénomène déjà dûment établi : Proust compose ses œuvres à partir de noyaux ${ }^{10}$ auxquels il fait des ajouts, ou qu'il juxtapose (les transitions étant exquisément subtiles, mais pouvant s'identifier, tel que l'on le signalera à sa place) les uns à côté des autres.

Au fait, cette dernière spécificité sera reliée des notions d'analogie dans l'ouvrage proustien, ainsi que de certaines techniques imitatives propres au pastiche (spécialement à celui qui nous occupe) dont le rapport cherchera à faire le point sur la question de la cohérence sémantique telle qu'elle sera amenée. Il s'en dégage manifestement que le procédé ne soit pas, à proprement parler, comparatif, car s'agissant de cet écrit particulier le rapport relève essentiellement, voire exclusivement d'une relation dite hypertextuelle ${ }^{11}$ et non pas comparative (le lien n'y étant pas forcément de parallélisme ou d'analogie, mais de dépendance). Mais il y reste ouverte la possibilité d'une étude ultérieure faisant la comparaison des deux situations créatives indiquées.

Quant aux enjeux esthétiques et éthiques de la voix narrative dans le pastiche de Saint-Simon par Proust, la question se veut plus simple (mais paradoxalement plus profonde et plus ample) : combien est-il pertinent de mener une étude narratologique ${ }^{12}$ dans un texte si particulier comme le pastiche en question ? Mais au-delà de la pertinence de la démarche, on s'est posé d'abord, avouons-le, une interrogation très basique : est-ce la même chose si lors de la narration, le narrateur est censé feindre être un autre écrivain (en l'occurrence, le duc Saint-Simon 
dans toutes ses particularités) que s'il doit seulement se constituer en narrateur fictif sans " aucun parangon réel " ? Il s'en découle clairement que l'on y trouve une différence fondamentale : la qualité de l'expérience esthétique (du côté de la voix narrative, rappelons-le) devrait être reconsidérée et analysée plus attentivement.

Au demeurant, l'expérience ici menée ne vise guère à dépasser l'état actuel de la discussion sur ce pastiche ni sur la série à laquelle il appartient, mais à élargir si possible le champ des considérations des phénomènes qui lui sont propres. Ayant une portée épistémologique qui se veut nécessairement restreinte (tellement les conditions de création et de réception de notre pastiche sont-elles spéciales), on craint d'ores et déjà qu'une transposition systématique, sur d'autres pastiches, ne soit guère valable, et qui plus est, qu'elle devienne une extrapolation incohérente. Ainsi peut-on penser que la réutilisation de cette structure logique, doit se faire, s'il en est besoin, sur des épreuves partielles et non totales. Somme toute, notre hypothèse cherche à donner de la voix, et peutêtre surtout, du visage (prenant l'avatar de ce pastiche), à une idée qui n'a été qu'esquissée de façon assez timide ${ }^{13}$ et qui mérite, nous paraît-il, que l'on lui accorde une place davantage notable dans le panorama des faits littéraires du pastiche L'Affaire Le Moine.

\section{B. État des lieux}

L’inconvénient de la "recherche ", c'est qu'à force de chercher, $i l$ arrive qu'on trouve... ce qu'on ne cherchait pas ${ }^{14}$.

Dès que l'on commence à fouiller à propos des études faites sur l'œuvre de Proust, surtout dans les relations que celle-ci entretient avec d'autres auteurs et ouvrages, on rencontre, tout de suite, des travaux portant sur les rapports d'influence stylistique et de réécriture ; c'est-à-dire qui relèvent des liens dits : intertextuels ${ }^{15}$. Ces travaux sont aussi ambitieux qu'ils sont beaucoup plus larges que celui que l'on envisage ici, puisqu'ils englobent le plus souvent tout l'ensemble d' À la Recherche du Temps Perdu, considéré comme l'œuvre-cathédrale ${ }^{16}$ de l'écrivain. On comprend, par là que cette thématique soit très susceptible de devenir le sujet et des mémoires et des études approfondies ${ }^{17}$, qui ne négligent point le lien sous-jacent de création entre Proust et Saint-Simon (pour certains il s'agit d'un rapport essentiel de modelage) ${ }^{18}$.

Outre cet axe de recherches si usité, il faut repérer, plus attentivement (puisqu'il y repose notre intérêt) les travaux portant sur l'hypertextualité chez Proust en général, et sur l'imitation ${ }^{19}$ que Proust met au point des " Mémoires de Saint-Simon " en particulier. On se doit, à cet égard, constituer un aperçu, quoique sommaire (car au-delà de leur mention, on ne s'attardera guère dans leur description) des travaux qui soient d'une façon ou d'une autre significatifs pour notre réflexion. On passera brièvement donc, tantôt par les ouvrages comportant le cadre théorique de l'étude, tantôt par les travaux mineurs, dont les articles qui contribuent dans des mesures différentes à nos effets spécifiques. 
Pour ce qui est de la théorie de la littérarité des rapports hypertextuels entre deux ou plus de textes, on doit forcément signaler l'ouvrage incontournable de Gérard Genette : Palimpsestes, sur les Éditions du Seuil. Le but monumental dudit travail consiste à établir un cadre de référence théorique fixé des divers avatars de l'hypertextualité et de les classer selon leur régime (fonction) et leur relation ${ }^{20}$ avec le texte; imitation (mimésis dira-t-il ${ }^{21}$ ) et transformation en grandes lignes, avec la panoplie des techniques qui en sont tributaires. Il serait aussi de notre intérêt le fait de signaler qu'une importante partie de la théorie y proposée est fondée et documentée sur les pastiches de Proust, dont celui auquel on se consacre, et qui constituent pour Genette de véritables objets exemplaires dans la collection des pastiches de la histoire littéraire globale.

Il en est un autre ouvrage dont aucune étude traitant l'activité pastichante chez Proust (et qui se veule sérieuse) peut s'en passer : Les Pastiches de Proust, édition critique et commentée par Jean Milly, chez Armand Colin. Un travail dont les efforts s'adressent à constituer un panorama exhaustif, fondamentalement dans le pôle de la création, des pastiches publiés dans Pastiches et Mélanges et quelques autres non publiés jusqu'alors. Milly en signale donc les parties composantes et les techniques mises en place, on y reviendra ; et établit quant à lui une démarcation provisoire du pastiche à partir des fonctions du langage proposées par Jakobson et Riffaterre ${ }^{22}$. C'est un travail méthodique de classement, qui se révèle fondamental pour toute étude génétique de l'œuvre proustienne, qu'elle se réduise aux pastiches ou qu'elle s'étende ailleurs.

Quant aux considérations sur les enjeux narratifs, toujours du côté du structuralisme, il nous faut signaler une autre œuvre de Gérard Genette, à savoir : Figures III aux Éditions du Seuil. Ouvrage qui vise à s'ériger comme une nouvelle poétique ${ }^{23} \mathrm{du}$ roman, précisément à partir de l'étude particulière et de la mise en place des théories sur À la Recherche du temps perdu (y regardé comme un unique et seul roman et non pas comme le titre générique de plusieurs romans). C'est l'un des piliers de la théorie narratologique structuraliste et la méthode qui s'y présente est censée pouvoir s'appliquer (enfin c'est l'un des ses objectifs manifestes) sur d'autres récits, à l'instar du pastiche de Saint-Simon, qui justement appartient au même auteur. Raison de plus, on le verra, pour le tenir largement en compte, bien qu'il soit du ressort d'un autre ouvrage.

Or, il en est en plus d'autres travaux mineurs qui on fait état des phénomènes directs ou adjacents dont il serait question ici. C'est le cas de quelques articles dont ceux des grands spécialistes du domaine : Jean Milly et Paul Aron que nous allons signaler en son temps. Nous procéderons alors à les regrouper, pour nos effets, en trois groupes relevant notamment du degré de filiation avec cette recherche.

En premier lieu, nous signalerons les articles « Proust : Philosophie du Pastiche " et " Pastiche de la Philosophie " publiés sur la Revue d'Histoire littéraire de la France et "Saint-Simon au miroir : trois pastiches des Mémoires aux XXe et XXIe siècles " sur Littératures Classiques, de Luc Fraisse et Florence MercierLeca respectivement. Les deux articles font le point, chacun à sa manière, sur quelques faits littéraires présents dans les pastiches de Marcel Proust. On en a 
soulevé particulièrement la "vocation épidéictique » ${ }^{24}$ (préalablement signalée) du texte pastichant Saint-Simon. Le premier vise, en principe et sans y entrer dans les détails, à établir un lien entre la technique créative mise au point par Proust dans ses pastiches et quelques courants philosophiques dont les systèmes de Kant et Leibniz ${ }^{25}$. Le second marque les tendances " interprétatives " que les différentes époques font du style saint-simonien, ou de ce qu'elles en conservent ${ }^{26}$ et en perpétuent; aspect plus que significatif pour nos propos.

Dans un deuxième temps, nous marquerons les études annoncées plus haut : "Les Pastiches littéraires dans À la Recherche du temps perdu " sur la Revue d'Histoire littéraire de la France et " Sur les pastiches de Proust : l'ethos et le champ ", sur le site internet de Contextes, toutes les deux de l'autorité de Paul Aron. Le premier portant sur les faits généraux de réécriture dans la $R e$ cherche, tantôt ceux dérivant de l'hypertextualité, tantôt ceux plutôt intertextuels, jusqu'à y faire même ressortir l'autopastiche ${ }^{27}$. L'autre posant son axe sur l'enjeu éthique dans la genèse des pastiches proustiens, à partir des valorisations que l'on en a faites, à l'époque où ceux-ci ont été créés. Aron en érige aussi une distinction essentielle dans ce sens :

Donc un ethos nouveau. Pour la première fois en effet un auteur en voie de légitimation exhibe cette part de la formation qu'est le pastiche comme une clé de sa compétence d'écrivain ${ }^{28}$.

\section{Cadre théorique et conceptuel}

Aimez donc la raison; que toujours vous écrits Empruntent d'elle seule et leur lustre et leur prix ${ }^{29}$

La cohérence tout entière des propos de cette étude repose, bel et bien, sur la compréhension que l'on a pu générer de quelques travaux du ressort de la théorie littéraire structuraliste. De ce fait, on a eu recours, par-dessus tout à deux ouvrages qui ont assez tôt fait le point dans leurs sujets respectifs, à l'intérieur de cette branche de la théorie littéraire. Le premier, occupant cette place tant il soutient les bases de notre réflexion (les définitions les plus essentielles on les y a empruntées), n'est autre que le grand traité sur l'hypertextualité : $P a$ limpsestes, de Gérard Genette. Le second, celui qui est censé donner la tournure particulière, on y aspire, à l'ensemble de cette considération est l'ouvrage, tout aussi genettien : Figures III. Travail dont l'objet est l'application d'une poétique du roman fixant son axe sur les faits de la narration.

Il se peut qu'ici, on se pose une question, d'ailleurs tout à fait valable : quelle intention d'employer et la théorie et la démarche structurales s'il y en a des plus récentes ? Cette interrogation n'étant point étrangère à nos réflexions, on en a un mot à dire. Décidément le structuralisme n'est pas le courant théorique le plus récent, et l'on pourrait aisément en trouver d'autres plus en accord (si cela existe) avec les tendances, pour ainsi les appeler, modernes. Mais une 
chose est certaine, le structuralisme littéraire s'avoue toujours valable et sensé, et son " âge " n'en détermine pas la pertinence, spécialement à l'égard de certains sujets dont le nôtre.

Puis il en est une autre raison, plus signifiante probablement, qui a fini par trancher de façon définitive notre choix. Outre, les grands mérites des travaux de Genette et la clarté de ses raisonnements, qui l'élèvent au rang de penseur au-delà de celui de critique littéraire, Gérard Genette est, rappelons-le, l'un des plus grands spécialistes de l'œuvre de Marcel Proust ; situation plus que favorable pour notre recherche. Ce n'est pas par hasard donc, que les deux ouvrages annoncés plus haut, prennent son élan des écrits de Proust. Palimpsestes (dans une moindre mesure par rapport à Figures III) établit maintes notions et définitions à partir des exemples tirés des pastiches de Proust, tel que l'on l'a dûment signalé. Quant à Figures III, sa démarche est complètement entamée sur les enjeux narratifs si particuliers de la Recherche ${ }^{30}$. C'est ainsi que dans le cadre stricte de notre recherche, cette concordance confère à ces ouvrages une condition heuristique irréfutable.

Or, juste avant de plonger dans le développement de nos propositions, on est obligé de dépenser un peu plus de temps à préciser quelques notions fondamentales pour la suite de notre étude. Tout aussi bien que des spécifications propres au texte traité qui devront y trouver leur place pour pouvoir être correctement saisies lors du développement argumentatif. Mis à part les deux ouvrages récemment mentionnés, on mettra à contribution, en plus dans ce compartiment, l'édition critique des pastiches de Proust par Jean Milly, ainsi que d'autres travaux mineurs convoqués selon le cas. On n'y vise pas à atteindre l'exhaustivité de la question, mais plutôt une précision suffisante qui nous permette de nos avancer d'un pas sûr.

C'est donc dans cet esprit que l'on passera d'emblée à tracer une limite sur les contenus narratologiques qui auront une certaine transcendance ici. Pourtant on y restera dans la surface, par l'instant, car tel que l'on l'a naguère annoncé, on réserve ces considérations pour une partie plus avancée du chemin réflexif. En conséquence, on est forcé de signaler comme strictement nécessaires, dans notre cas, les faits suivants de la narratologie : ordre (dans une moindre mesure), c'est-à-dire en termes généraux, les questions du temps, mode (degré de " participation " du narrateur) et voix narrative (rapports entre les différents degrés des narrations, " les narrateurs » et leurs récits) qui est certainement celle qu'il y a de plus significative à notre égard.

On laisse donc de côté les autres aspects entretenus par Genette dans son travail, à savoir : durée et fréquence. Puisque l'on considère qu'ils n'apportent guère ici, eu égard à la perspective prise qui relève plutôt des enjeux d'identité du narrateur-auteur, du décalage temporel narratif et de l'implication du narrateur dans son récit. Et puis il faut s'apercevoir du fait de la proximité que les études sur l'imitation stylistique (dont on se sert aussi, bien entendu) ont avec nos deux sujets majeurs. 


\section{C.1. Définition du terme pastiche}

L'édifice de nos arguments requiert à ce que l'on s'applique désormais à la définition, et qui plus est, à la confrontation des définitions concernant la pratique du pastiche et davantage du pastichisme proustien. On devra toujours prévenir que l'objectif n'y est point celui de nous inscrire dans la discussion générique du pastiche, mais tel que l'on l'a assez tôt remarqué : faire état des notions essentiels pour nos propos et échafauder par la suite des bases solides pour le reste du travail. Il s'en suit, et pour cause, que l'on ne cherchera à présenter des définitions catégoriques parfaitement démarquées dans le texte. La suite des arguments répond pourtant à une démarche logique qui vise à faire avancer les thèmes le plus convenablement possible.

Commençons aussitôt par cerner le concept du pastiche. Étymologiquement parlant Genette en fait le point :

Le terme de pastiche apparaît en France à la fin du XVIIIe siècle dans le vocabulaire de la peinture. C'est un calque de l'italien pasticcio, littéralement " pâté ", qui désigne d'abord un mélange d'imitations diverses, puis une imitation singulière. En 1767, Diderot (...) parle de son équivalent littéraire (...) comme d'un genre possible ${ }^{31}$.

On comprend immédiatement que cette acception fait référence à une classe de pratique artistique, relevant jusqu'à là de deux techniques précises : l'imitation singulière, et l'imitation conjointe (le mélange n'étant que le bon agencement de la juxtaposition de plusieurs imitations singulières). Néanmoins, il est nécessaire de remarquer que lorsque Diderot s'y met à réfléchir, selon Genette, le mot genre fait son apparition ${ }^{32}$. Tel est en somme (résumé peut-être brutal) l'état actuel de la définition du pastiche : tantôt pratique, tantôt genre. En ce sens, on doit se rapprocher de l'une des plus brillantes réflexions faites à ce titre. Genette propose une relation d'analogie, qui éclaircit assez largement le problème pour nos effets, dans laquelle l'imitation est, en grandes lignes, la technique (" figure ") centrale du pastiche. Pour lui, il ne s'agit guère des formes et figures particulières présentes dans un pastiche, mais du fait qu'elles sont empruntées voire copiées ; le style tout entier d'un écrivain y devenant une forme imitable. Ainsi l'on y établit le rapport suivant:

imitation : rhétorique :: pastiche : poétique..$^{33}$

Ce schéma analogique tiendra une application plus qu'évidente dans la suite de nos commentaires. C'est peut-être le résumé théorique le plus précis auquel on peut avoir recours. Nous pourrions ajouter en amont maints exemples s'inscrivant dans cette perspective, nous en citerons un de l'autorité de Paul Aron : « Le pastiche peut-être défini comme l'imitation (...) du style d'un auteur ou d'un courant littéraire ${ }^{34} »$. On octroie alors à l'imitation le rang d'axe central dans l'étude de l'activité pastichante, notre démarche en est aussi tributaire, à 
l'exception près, que l'on envisage les implications de l'imitation narrative en particulier. Milly de son côté essaie d'instaurer une définition méthodologique du pastiche, ayant pour parangon précisément les pastiches de Proust. Son procédé se sert des études en linguistique, tant il fonde son intervention sur les fonctions du langage établies par Jakobson et Riffaterre.

Une démarcation s'y fait remarquer tout de suite, pour Milly le pastiche se différencie des autres genres car « le donné qu'il organise est déjà lui-même organisé " et par conséquent il présente un " contenu [qui] est (...) contenant-contenu $(. . .)^{35}$ ». Pour lui donc trois fonctions se partagent la plupart du champ, soit : "référentielle, métalinguistique et conative. " La première y est censée connaître deux référents : le thème traité et l'œuvre imitée ; la deuxième, rapportée spécialement au second référent ${ }^{36}$, est la plus proche de l'imitation dont nous venons de parler car c'est celle qui est chargée de copier ce qu'il y a de particulier dans le texte imité. La troisième, on le sait bien, gère les effets que le texte (en tant que message) veut produire sur le public récepteur. Ceci, dans le cas particulier qui nous occupe doit se relier à la vocation épidéictique du texte, dérivant de l'effet social que Proust veut manifestement produire avec ce pastiche.

Ensuite Milly constitue un lien de complémentarité entre le pastiche et la critique littéraire, à partir des fonctions métalinguistique et expressive (conative). La fonction conative s'imposant le plus souvent dans les pastiches, tandis que dans la critique, c'est à l'inverse : la fonction métalinguistique y est dominante. Voici la synthèse qu'il en fait :

Critique et pastiche se trouvent être ainsi, du point de vue de cette fonction [métalinguistique], des genres complémentaires. De plus le terme de métalinguistique doit être pris dans une acception large, (...) rhétorique et idéologique : (...) le pastiche permet à Proust de dégager (...) les formes d'expression, [et] (...) les formes de contenu (...) de ses modèles ${ }^{37}$.

Idée plus que significative et ayant une filiation directe avec la conception que Proust lui-même érige de son travail de pasticheur. Tel qu'en témoigne cet extrait d'une lettre de Proust, recueillie par Paul Aron, selon lequel l'auteur entendait présenter ses pastiches :

(...) avec des études critiques parallèles sur les mêmes écrivains, les études annonçant d'une façon analytique ce que les pastiches figuraient instinctivement (et vice-versa), sans donner la priorité ni à l'intelligence qui explique, ni à l'instinct qui reproduit ${ }^{38}$.

Encore, peut-on relever une autre similarité existante entre le pastiche et la critique, et c'est celle qui a été signalée par Genette à propos du rôle du structuralisme au sein de la critique littéraire. C'est ce caractère qui l'approche du « bricolage $»^{39}$ et que si l'on s'aperçoit est partagée par l'imitation du pastiche, tel que la mention préalable des fonctions du langage l'a laissé entrevoir : le pastiche, 
aussi bien que la critique, ne dispose que des éléments tirés du cadre restreint du texte source, dans son cas, nommé l'hypotexte.

Une autre " issue » du fait de la place centrale de l'imitation dans le pastiche est la reconnaissance, qui se gère chez le lecteur, de la « langue d'auteur ${ }^{40}$ ». Ceci est l'un des faits les plus importants que l'on ait signalé jusqu'à ce point, en ce que le pastiche comporte deux niveaux essentiels de lecture (lecture superficielle de l'hypertexte et reconnaissance de l'hypotexte). Alors que la plupart des autres genres littéraires n'en ont besoin que d'un pour que le lecteur y parvienne à saisir leur sens basique. Il faut admettre que la mise au point de cette " condition de lecture ${ }^{41}$ " implique un jeu d'alternance continuelle et subtile "d'identités " (perceptibles d'un côté dans les anachronismes et de l'autre dans les emprunts directs au modèle) que doit y assumer le narrateur. Sa tâche consistant à faire ressortir la narration d'un autre, mais de façon à ce que l'on puisse toujours reconnaître qu'il s'agit d'un texte imitatif et non pas le prendre, à tort, pour un originel de l'écrivain imité. L'art s'y opérant sous le bon agencement des effets de la similarité et de la différence, on y reviendra plus tard.

À l'intérieur de la plus qu'ancienne discussion littéraire entre mimésis et diégésis, on rencontre un jalon qui nous sera utile pour achever de cerner la notion du pastiche autour de l'imitation comme son trait principal. En ce sens, selon Barthes, en parlant du récit, l'imitation n'est pas nécessaire mais contingente ; la fonction du récit n'étant point mimétique et le fonds résidant dans la logique qui s'y expose ${ }^{42}$. Le pastiche est sans aucun doute l'une des formes du récit, quoiqu'il ne soit, et pour cause, compris dans cette caractérisation générale. Disons pour l'instant que le pastiche en serait le cas exceptionnel, puisque sa cohérence interne est déterminée par le rôle qu'y tient l'imitation. Et même quelque logique apparente qu'il puisse présenter ne serait-ce que l'emprunt d'une logique étrangère ou le cas échéant, comme celui de Proust : une logique analogue à celle du modèle ; la véritable logique du pastiche étant, de toute façon, celle de maîtriser, nous y insistons à nouveau, la technique artistique de l'imitation et son effet (signe de réussite) de ressemblance.

\section{Spécification du pastiche L'Affaire Le Moine}

Sans avoir été exhaustifs, il nous semble que l'on a bien délimité la notion du pastiche, du moins ne serait-ce que du côté de nos plus stricts intérêts. Le mouvement logique de notre exposé nous exige désormais passer du général au spécifique. Aussi, l'on va tâcher de fournir un aperçu suffisant des particularités relevables des pastiches de Marcel Proust et surtout, par des raisons évidentes, de celui de Saint-Simon. En tout cas, comme il sera signalé par la suite, ce pastiche présente nombre de spécificités que les autres ignorent quant à eux ; la longueur (29 pages pour celui de Saint-Simon, 11 pour celui de Renan, et environ 5 pages pour chacun du reste) n'en est que l'une des caractéristiques les plus perceptibles. Proust y a mis de soi, il n'a pas uniquement voulu pratiquer un exercice artistique littéraire, il y a passé un message pour sa " société » dans un 
code (celui de Saint-Simon, et par son biais celui de l'ancienne cour) qui serait transcendantal pour cette " société ".

Les pastiches composés par Proust, d'emblée, appartiennent à une époque où cette manifestation artistique foisonnait, voire elle était en vogue, tel qu'en témoigne Milly dans son Historique des pastiches "Lemoine» ${ }^{43}$. Cela implique que de la part du public récepteur, il doit forcément y exister une sensibilité accrue par rapport aux récits pastichants. Il s'agit en somme d'une forme de divertissement élitiste, voire d'humour, dépendant du cas. Le pastiche de SaintSimon a, à ce titre, un public cible assez restreint, c'est le cercle social de Proust qui est visé et atteint, tant nombre d'entre eux y sont inclus, soit pour leur dédier un éloge, soit pour les blâmer à cause de leur conduite sociale (faut-il rappeler à nouveau la fonction épidéictique si importante de ce texte ?). L'ensemble de ces enjeux éthiques sera considéré ailleurs, ce qui nous intéresse ici, c'est le fait de constater combien cette forme du pastiche, si « spécifique " pour ainsi dire, relève d'un processus de raffinement de ce spectacle. De part et d'autre, autant auteur (création) que public (réception) ont dû se soumettre à une certaine évolution, c'est-à-dire une sophistication pour passer de l'imitation en termes drolatiques, ludiques ou esthétique-artistiques à une imitation, que tout en restant vraisemblable, comporte un message didactique.

Un autre aspect à saisir, cette fois-ci du côté de la stylistique des pastiches, c'est l'idée que Proust lui-même en avait à cet égard. Avançons donc par étapes. À un moment donné de son exposé sur l'imitation, Genette ${ }^{44}$ décide qu'il faut changer de terme : il s'y infléchit donc pour le mot : "mimétisme ", car pour lui celui-ci s'approche davantage (dans sa désinence en -isme) aux "idiolectes" propres au style de chaque auteur, dans notre cas, il serait alors question des " saint-simonismes " (étiquète collective regroupant les traits particuliers de l'écriture de Saint-Simon). Stylistiquement parlant, on aura affaire à deux procédés différents et complémentaires : d'abord la reconnaissance et le ramassage des traits les plus distinctifs (il faut rappeler ici que ce choix dépend largement de la tournure que le pasticheur veut imprimer dans son pastiche et de la perspective qu'il accorde à la stylistique imitée), et puis de la mise en œuvre de leur imitation (ou mimétisme) dans le texte pastichant. En d'autres termes : " analyse et synthèse ${ }^{45}$ " tel que l'a posé Mercier-Leca.

Proust, quant à lui, en était largement conscient. Si bien qu'il a contribué avec une notion fondamentale pour la théorie du mimétisme. Les "phrases types ${ }^{46}$ ", seraient donc des unités, bien entendu au niveau phrastique de la langue, qui caractérisent l'écriture de chaque écrivain en particulier. Une " phrase type » est censée contenir plusieurs "idiolectes ", elle est, tout bien considéré, le traitement juste et la répartition adéquate des "idiolectes " selon le modèle choisi. Bref, c'est la structure minimale à retenir et pour la technique du pastiche et pour les effets qui s'y cherchent. Proust laisse voir par là, qu'au moins en ce qui lui concerne, lui, il exécute son opération au niveau de la phrase et que le choix de cette dénomination n'est pas accidentel. Ceci est d'une immense valeur heuristique pour les considérations que nous allons développer dans la section consacrée à la cohérence sémantique. De toute façon, cette notion mise au point par l'auteur du 
pastiche L'Affaire Le Moine, marque une norme prescriptive dans le courant de l'imitation pastichante, tout comme l'a fait à son époque et dans le champ de la création des personnages romanesques, la notion des "personnages type $"{ }^{47}$.

Il en est un trait qui représente de manière assez patente ce dont on vient de parler, et qui plus est, au sein du pastiche de Saint-Simon. C'est l'utilisation des " embrayeurs" ou en d'autres mots le recours à la " deixis ". En fait, une lecture sommaire du pastiche est supposée de relever maints exemples du type : " qui a été marqué en son lieu ", " dont a déjà été parlé ", " comme on l'a vu ", " il a été ici trop souvent parlé de lui en son temps ", "il a été marqué en son temps ", " on le verra par la suite», " je me suis assez étendu en son lieu » et ainsi de suite. Ces exemples renvoyant tantôt au passé, tantôt au futur interne du texte, sont le résultat d'un procédé mimétique tiré de leur aussi large présence dans les Mémoires, sauf qu'ici ils ne renvoient à aucun référent ou contenu existant mais à un effet d'illusion.

En toute vérité une lecture superficielle, voire naïve, croirait en ce que le pastiche est " inséré " quelque part dans les Mémoires, c'est dire que l'on pourrait repérer peu ou prou, à l'intérieur de celles-ci, le moment qui revient à la séquence fictive du pastiche. Certes, on peut délimiter une grande section où l'on pourrait " situer " cette mémoire fictive (mis à part la présence fondamentale des anachronismes), cette section se retrouverait, en grandes lignes : après les morts de Monseigneur le Grand Dauphin et du roi Louis XIV, mais il y est question, rappelons-le, d'un référent illusoire. C'est ainsi que la deixis du texte originel prolonge son ombre sur l'hypertexte pour y retrouver l'aspect de fils qui soutiennent sa couture : mirage réussi. Une telle particularité renvoie forcément aux caractéristiques du genre imité, et quoique le courant général des études sur les pastiches négligent l'importance, voire la pertinence de trop repérer dans l'hypotexte à proprement parler, nous allons de notre côté remarquer certains faits qui appartiennent directement à l'hypotexte et qui selon nous affectent l'avatar hypertextuel des façons très spécifiques, dont celle qui vient d'être exposée.

Encore nous faut-il considérer deux particularités attribuables au pastiche de Saint-Simon. De manière générale, Proust avoue éprouver de l'horreur du pastiche " involontaire ${ }^{48}$, toute imitation serait pour lui consciente, maîtrisée, décomposée et réintégrée à volonté. Tant s'en faut pour que ce procédé soit regardé comme un automatisme. C'est-à-dire que la "vraisemblance " (au second degré : que le pastiche tienne suffisamment l'aspect d'un écrit des Mémoires) du pastiche en question répond à une démarche méditée et son résultat n'est donc point accidentel. À cet égard Genette écrit :

Le pastiche, dont la fonction est d'imiter la lettre, met son point d'honneur à lui devoir littéralement le moins possible. La citation brute, ou emprunt, n'y a point sa place ${ }^{49}$.

L'autre aspect à en retenir, et qui s'y ajoute en amont, c'est le caractère d'hommage $^{50} \mathrm{du}$ pastiche de Saint-Simon par Proust. En effet, l'intention n'y est absolument pas celle de se moquer de l'auteur des Mémoires, ni de son style. 
On verra qu'il se sert plutôt de ce ton grave et péremptoire propre au duc, pour attaquer d'autres cibles extérieures au texte. Malgré ce respect solennel, une petite critique s'y laisse percevoir : dans la toute dernière phrase du pastiche Proust (est-ce lui ? d'où notre intérêt pour les enjeux narratifs y compris !) plaisante d'un ton dissimulé sur les obsessions de Saint-Simon qui se reflètent dans le style de son écriture : " Mais cette disgression sur les titres singuliers nous a entraînés trop loin de l'affaire du Moine ${ }^{51}$. " Proust en tire du profit pour justifier les déviations et thématiques et sémantiques (comme on le verra plus tard) et pour laisser ouverte la possibilité d'une suite. Ainsi le rapport éthique entre Proust et son modèle peut très bien se définir comme un mélange « d'admiration et d'ironie ${ }^{52}$ ".

\section{D.1. Les techniques du mimétisme proustien}

En ce moment de notre exposé, il nous semble cohérent d'envisager plus de précision dans la terminologie stylistique, et de ne plus se contenter avec une mention sommaire des effets imitatifs. Or, nous allons nous restreindre aux techniques significatives pour l'analyse du pastiche de Saint-Simon, et peut-être surtout, pour les thèses que l'on essaie de soutenir, quitte à tromper les expectatives amorcées par le sous-titre généralisateur de cette partie. On devra par la suite délaisser, hélas !, certains aspects techniques qui ne contribueront pas au progrès des réflexions entreprises ; plus on devient spécifique, on le sait tous, plus d'idées on est forcé de négliger.

Par-dessus tout, il est essentiel de commencer en signalant les facteurs dont le rapport soutient l'équilibre tout entier de la structure de l'œuvre. Nous les avions déjà convoqués lorsqu'il était question de l'art de l'imitation dans le pastiche. Rappelons-le : le pastiche doit être assez similaire au texte source pour se considérer réussi dans ses propres termes, mais il doit, tout en même temps, étaler son identité imitative, faute de quoi il risque de se faire confondre avec un texte originel de l'auteur imité, ou tout au moins à une copie ratée ou automatique, sans aucun effet (ludique, satirique, artistique ou didactique) appréciable de la part du lecteur. C'est finalement l'équilibre entre la ressemblance et la différence, idée qui est très chère dans la création artistique proustienne, tel qui le montre Genette $^{53}$, pour qui le jeu et "l'essence " de la métaphore chez Proust dans la Recherche reposent, de fond en comble, sur cette relation ponctuelle. Milly quant à lui aussi, s'est bien rendu compte de cette dynamique, au cœur des pastiches :

L'expressivité principale et la force comique tiennent à ce que le lecteur perçoit, simultanément ou successivement, une ressemblance et une différence avec le modèle ${ }^{54}$.

Ce point arrivé, on se doit de signaler quelques exemples précis de ce traitement de "l'essence " du texte : la "ressemblance-différence " (point d'équilibre de la technique imitative, dont le dosage à mauvais escient déborderait 
sur "l'engluement" ou "la dissonance" selon le cas ${ }^{55}$ ). Or, il est vrai que dans le texte en question abondent les exemples de ce type, pourtant on n'entend pas à épuiser la démonstration sur ce point, et l'on va se contraindre d'en citer quelques-uns. Seulement à l'intérieur même du portrait du duc d'Orléans on peut relever plusieurs fois l'application technique de cette balance. Voyons donc que presque tout ce qui s'y dit de lui est "vrai » (c'est dire que c'est effectivement présent dans les Mémoires), mais arrêtons-nous sur un point particulier : son goût pour la chimie ${ }^{56}$ (entendre : alchimie) ; pierre angulaire de la connexion hypertextuel (faut-il remarquer que c'est par ce biais que Proust parvient à " introduire » l'Affaire Lemoine dans la cour du Régent, et qu'elle s'y transforme en Affaire Le Moine ?).

Ce trait étant donc présent, tantôt dans l'hypotexte, tantôt, évidemment, dans l'hypertexte ; on y a affaire à une ressemblance patente. Il y aurait alors, faute d'une lecture approfondie de l'hypotexte, une tendance à croire que seul ce pôle du rapport équilibrant s'y manifeste. Néanmoins quelque part dans les $M e ́-$ moires on rencontre un témoignage divergent : le Régent se moque de la fabrication d'or ${ }^{57}$. Cela soulève un questionnement, pour ainsi dire, la ressemblance de la fiction à la source devient par conséquent sujet d'interrogation (enjeu typique de la vraisemblance littéraire). Certes le Régent (comme personnage fictif d'un texte littéraire, on n'a pas affaire ici à l'histoire) aimait l'alchimie, ce qui était mal vu dans le monde de la cour (et surtout dans le récit du narrateur), mais cela n'implique pas (la preuve en est à la main) qu'il croirait à tout et à n'importe quoi que l'on lui proposait d'alchimie. La tâche y est accomplie, l'imitation est vraisemblable : souvenons-nous que la place qui revient à la vraisemblance est celle de la plausibilité et non pas celle de la certitude.

Toujours dans le repérage des exemples de la "ressemblance-différence ", il est ensuite un autre cas assez intéressant. Le rapport éthique " des narrateurs » avec les Noailles ${ }^{58}$. Ce qui en est curieux, c'est le fait que cet anachronisme est fondé sur la parenté effective de ces personnages, en réalité, la femme appartenant à la famille Noailles dont parle Proust descendait des Noailles apparaissant dans les Mémoires et auxquels Saint-Simon n'accordait aucune bienveillance. Cet exemple s'inscrit, d'ailleurs, dans la perspective de la vocation épidéictique du texte, vocation partagée par le texte source. Mais il renvoie aussi à une notre dénomination, et peut-être avec une autre nuance, du paradoxe d'équilibre " ressemblance-différence ", c'est le rapport " dépendance-indépendance " entretenu par les textes. La nuance qui les sépare est tout simplement qu'ici le référent du paradoxe n'est pas fictif (ni stylistique) mais il peut se retrouver dans le monde factuel. Cela confère au texte imitatif une certaine liberté dans sa portée (qui doit pourtant être toujours justifiée, tel qu'il l'est dans ce cas) vis-à-vis du texte imité.

En outre, on constate par là, combien le portrait est un composant majeur du pastiche L'Affaire Le Moine, puisqu'il l'est tout aussi du texte source. Alors trois procédés techniques sont à retenir en ce sens, Mercier-Leca les a baptisés selon les "termes freudiens : dissémination, condensation et déplacement " ${ }^{59}$. Le premier consiste à redistribuer les traits d'un personnage (que ce soit du côté de l'éthopée ou de la prosopographie) sur plusieurs autres dans l'hypertexte. Le deuxième est 
purement le procédé inverse, et le troisième est le fait d'octroyer à un personnage de l'hypertexte une caractéristique propre à un personnage de l'hypotexte (on y requiert, naturellement, que tous les deux personnages soient présents dans les deux textes). L'analyse de ces procédés sera très importante, voire précieuse pour le développement des idées touchant à la cohérence sémantique et notamment à son compartiment à propos de la thèse de création proustienne à partir des noyaux sémantiques. Nous y réserverons les exemples pour cette raison.

Cependant pas toutes les lectures du pastiche de Saint-Simon y repèrent les mêmes techniques. C'est le cas de la « saturation ${ }^{60}$ " qui n'est pas universellement admise comme manifeste dans ce pastiche. En termes généraux, on en a rendu compte préalablement, le pastiche de Saint-Simon par Proust est regardé plutôt comme un hommage que comme une moquerie. On établit cette différentiation car la "saturation " est proche de la caricature en ce qu'elle exagère et intensifie les traits distinctifs d'un style par le moyen de l'itération (selon Milly ceci relève d'un procédé synecdochique ${ }^{61}$ ). Elle est adressée pour autant dans la direction de la raillerie ou de l'ironie, qui est presque exclue de l'interprétation critique de ce pastiche, on dit presque parce que nous-mêmes, nous avons signalé un certain esprit d'humour taquin qui pourrait se reconnaître dans la fin du pastiche, ou au moins que cette fin en avouerait la présence.

Il en est des critiques qui ont un avis moins modéré sur ce point et qui y voient même du burlesque, dont celui de D.C. Cabeen :

«In it [dans le pastiche] Proust brings out-exaggerated in places almost to the point of burlesque-the well-known qualities of the Duke: his insatiable curiosity for gossipy anecdote, his vanity of a grand seigneur, his detestation of the rising bourgeoisie, his preoccupation with questions of procedure and court etiquette, his penetrating eye for characteristic human traits as revealed by peculiar mannerisms, $(. . .)^{62} »$.

Mais jusqu'où arrive véritablement cet humour, si Proust même fait un reproche dont nous ne pouvons pas ignorer les échos ? : "Le pastiche fait sourire. Même avec le plus tendre respect, il ne faut pas sourire de ce qu'on aime ${ }^{63}$. Et il assez probable que c'était de l'amour ce que Proust éprouvait pour les Mémoires de Saint-Simon, telle serait en tout état de cause notre position.

En dernière analyse, en ce qui correspond aux techniques mimétiques mises en place dans le pastiche, nous en retrouvons une qui est plus en accord avec l'esprit d'hommage. C'est la création autonome par rapport au modèle, quoiqu'il semble paradoxal de parler de création ou d'invention au sein d'une pratique gérée par l'imitation. Pourtant le progrès logique de notre réflexion a suffisamment balisé le terrain pour que l'on puisse traiter cette forme extrême, bien que toujours équilibrée, de la tension " ressemblance-différence ", qui s’infléchit souplement du côté de la différence tout à la fois qu'elle est accrochée, par un fils tendu et minimal, à la ressemblance.

Dans le cas du pastiche de Saint-Simon, il y a un mot qui n'est pas tout à fait copié de son hypotexte, c'est Proust qui décide de l'y mettre: " marli » ${ }^{64}$. Nous 
avancerons, une possible thèse à son égard. Ce " hapax " (en réalité il y apparaît une fois sous sa forme pluriel et puis derechef dans sa forme singulière) est une nouvelle forme tiré du toponymique : "Marly ". Dans le lexique du duc de SaintSimon, ce mot pouvait signifier soit le château à Marly, soit par métonymie les réunions sociales qui s'y déroulaient (nous le retrouvons une fois dans ce sens ${ }^{65}$ ), et encore par synecdoque, certaines réunions du même type tenues ailleurs. Postérieurement le mot " marli » désigne en français un tissu fin ou le bord décoré d'une assiette, apparemment associant (métonymiquement à nouveau) le luxe de ces articles au luxe dudit château ${ }^{66}$. Ainsi le mot de Proust est un hybride des deux formes, et si l'on croit en leur rapport étymologique : de deux graphies. Du premier, chronologiquement parlant, il en conserve un sens, soit : celui de réunion sociale de la cour, du second il gardera la graphie.

Ce procédé par lequel l'auteur se détache légèrement du style imité par une production qui pourtant s'en inspire, doit forcément nos rappeler l'ancienne innutrition. Quoique l'innutrition ne se posât pas en termes d'imitation, elle prônait le dépassement du texte inspirateur par le moyen du texte hommage (notion partagée avec ce pastiche, ou du moins avec l'interprétation que nous en faisons) rédigé en style similaire. Deux citations peut-être seront-elles susceptibles de nous en apercevoir. D’abord Milly par rapport à la création des pastiches :

L'activité métalinguistique du pasticheur peut donc être telle qu'après avoir assimilé les structures originales de son modèle, il est capable de prendre à son tour une attitude créatrice analogue ${ }^{67}$.

Ensuite Genette, en citant Proust tire la réflexion suivante, qui s'ajuste parfaitement à la nôtre : « «(...) on ne peut refaire ce qu'on aime qu'en le renonçant», c'est-à-dire ici en le dépassant. ${ }^{68}$ " C'est comme le dit Mercier-Leca, en termes psychanalytiques : l'assassinat symbolique du modèle-père ${ }^{69}$. Peut-être ce " dépassement » (qui ne s'exerce point en termes de supériorité esthétique, mais d'hommage et d'indépendance vis-à-vis de la source d'inspiration) ne s'opère exclusivement dans le pastiche mais il faudrait y envisager aussi À la Recherche du temps perdu et les études d'intertextualité y retrouveraient ainsi, à notre égard, un nouvel intérêt.

\section{E. La cohérence sémantique}

On propose ce terme en fonction des opérations analytiques que nous allons développer dans le texte. Lesdites opérations auront lieu dans deux degrés différents de la littérature, chacun regroupant à son tour, on le verra, plusieurs niveaux du texte. C'est ainsi que l'on travaillera, en grandes lignes, sur la genèse artistique du texte, perspective proche de la génétique littéraire et puis sur « le résultat " de ce procédé sur l'aspect final du texte, soit la dispositio et l'elocutio rhétoriques. Précisons donc le terme : la notion de cohérence sémantique repose sur un raisonnement d'unité lexicale et de sens (sémantique), en termes rhétoriques nous dirons qu'il s'agit d'un rapport de contiguïté, alors métonymique 
qui gère la cohésion interne des composants d'une partie : des mots si l'on est au niveau de la phrase, des phrases si l'on est au niveau des paragraphes, enfin des paragraphes s'il s'agit des grandes parties du texte. Nous allons quant à nous rester plutôt dans les deux derniers rapports : composants-niveaux.

Jusqu'ici il est clair qu'il s'agit d'une perspective qui privilégie l'observation de l'unité sémantique interne des parties. Mais dès que l'on parle de cohérence c'est qu'il doit y avoir quelque parangon extérieur qui remplit la fonction prescriptive déterminant ce qui est cohérent ou pas. Ce n'est certes pas la cohérence des composants internes les uns par rapport aux autres, par la toute simple raison qu'une logique pareille exigerait à ce que l'on privilégie l'un des composants sur les autres, suivant très probablement un critère subjectif. À quoi doivent répondre donc et la sémantique et l'organisation du texte ? Il s'agit en principe de retracer "l'intention " désignée par l'auteur à chacune de ces parties. Or, comment une chose pareille serait-elle possible ? Heureusement il est un composant typique de l'œuvre saint-simonienne que Proust a décidé de garder : les manchettes. Ce sont bien elles les parangons de cohérence correspondants aux grandes parties du texte, on y reviendra bientôt.

D'après le schéma que l'on vient de proposer, on doit d'abord se concentrer dans l'aspect de la genèse du texte. Il est une caractéristique de la création proustienne qui parmi toutes les autres est susceptible d'être analysée en ces termes. Les études de la syntaxe et du style de Proust ont suggéré la thèse selon laquelle, l'écrivain travaillait à partir des noyaux auxquels il ajoutait des accessoires, pour ainsi dire, ou encore celle de la composition mosaïque par fragments des plusieurs rédactions, tel que l'a remarqué Milly ${ }^{70}$. Ce procédé qu'il s'opère dans la phrase ou dans une partie plus large, est purement métonymique, comme nous l'avons déjà marqué. Il nous fait penser à cette comparaison (qui peut et qui va d'ailleurs se prouver dans le texte) : les noyaux sémantiques (puisqu'il y est question de rapprochement du sens) fonctionnent comme des " aimants " qui convoquent à l'esprit de Proust tout ce qui est proche (et utile !) à l'idée qu'il est en train de traiter.

Les manchettes, à nouveau, nous procurent précisément par rapport au pastiche, une notion approximée de ce qui avait Proust à l'esprit. Ce qui s'en écarte, c'est-à-dire les déviations sémantiques, répondent tout aussi à ses intentions ; il est juste nécessaire de les saisir correctement et d'en interroger la cause. Peut-être l'avons-nous déjà signalé : il s'agit pour la plupart du temps, de l'inclusion d'un portrait anachronique à fonction épidéictique ou encore d'un effet d'imitation des déviations ou des allongements " excessifs" présents dans les textes saint-simoniens. En tout état de cause, et c'est là le génie proustien tout entier, la forme qu'il choisit pour " se dévier " en y incluant ces anachronismes, c'est le portrait, forme plus que maîtrisée par Saint-Simon, et par ce fait ils relèvent toujours de l'imitation. L'équilibre : "ressemblance-différence " s'y maintient intact, puisque derechef, ce qui ne dérive que de la verve de Proust est agencé de façon à ne pas perdre le contact avec l'hypotexte.

D'après le renseignement qui nous en pourvoit Jean Milly, le pastiche est composé de deux grands noyaux, en tant que rédactions différentes à l'origine du 
texte final, à savoir : "Fête chez Montesquiou à Neuilly " et "l'épisode Murat " " Tous les deux, curieusement, sont à l'origine de la vocation/intension épidéictique du texte, ils se retrouveront, chacun à sa façon, des déviations sémantiques à l'intérieur de notre analyse. L'épisode Murat est étalé dans les manchettes, et sa déviation comporte l'insertion d'un sujet au milieu d'un autre. Le portrait de Montesquiou, de son côté, ne figure pas dans les manchettes et sa déviation peut se regarder, pour l'instant, comme très accentuée. Pour Milly cette situation retrouve un reflet direct dans le texte : " D'où provient fréquemment, dans les pastiches définitifs, une impression de coq à l'âne qui contribue au comique, mais n'est pas un élément emprunté $»^{72}$.

Or, si pour Milly, cette déviation n'est pas un emprunt (une imitation), elle doit, du moins dans le cas du pastiche de Saint-Simon, comporter une coïncidence perçue par Proust. Probablement l'exemple le plus relevable que l'on peut mentionner à ce titre, est le passage sur le mariage de Delaire ${ }^{73}$ qui est inséré juste après les commentaires correspondant "Chimères des Murat sur le rang de prince étranger " (l'un des sujets les plus importants du pastiche) et avant l'étalage des personnages assistant au "parvulo de Saint-Cloud". La raison de son inclusion dans ce lieu précis ne vient que jusqu'au paragraphe suivant et très subtilement pour introduire un autre mariage : «Un autre et plus grand mariage retarda la venue du roi d'Angleterre $(. . .)^{74}$ »

On en dégage encore que les mouvements (qu'ils soient de déviation ou de retour sur le sujet) sont délicatement justifiés et par là même liés (sémantiquement) à l'ensemble du texte et à la suite des arguments qui le comportent. Ainsi le portrait de Robert Montesquiou-Fézensac (composant transcendantal du texte, pour certains y réside même " la perfection ${ }^{75}$ de ce pastiche par rapport aux autres) est introduit par le biais d'une parenté (avec d'Artagnan) qui déclenche un grand développement où il se trouvent inclus, à son tour, d'autres portraits. Cette " déviation ", peut-être la plus longue que l'on peut y repérer, convoque à nouveau, une réflexion sur la métonymie, car c'est elle qui gère les techniques de rapprochement sémantique par le moyen de ces petits " ponts " entre les parties du texte. Ici, la métonymie tient la place de privilège dans la création, en raison de la nature du texte imité. Notamment parce que la métaphore selon Genette y était chassée " par l'objectivisme de l'ethos classique ${ }^{76}$ ». Proust est forcé, par conséquent d'y adapter sa verve littéraire.

C'est précisément cette adaptation celle que nous proposons comme la responsable de la notion (métaphorique) des " aimants sémantiques", simple description de notre compréhension sur la démarche créative proustienne dans ce pastiche. Référons-nous à un exemple concret : lorsqu'il y est question de Robert Montesquiou, on vient de le dire, d'autres portraits sont développés, aussi rappelons-nous de celui d'Yturri ${ }^{77}$, "l'ami espagnol de Montesquiou ». Dans le monde réel Yturri, secrétaire de Montesquiou, était $\operatorname{argentin}^{78}$, or la mention d'un argentin n'aurait pas de lieu chez Saint-Simon. Proust décide alors de lui octroyer la nationalité espagnole dont il partage au moins la langue, et d'un seul coup il y relie l'ambassade de Saint-Simon à Madrid. Ou sinon, il y a le cas, plus simple du comte de Stanhope, secrétaire d'État d'Angleterre chez Saint-Simon, et nom 
fictif du roi d'Angleterre pour passer inaperçu en France, dans le pastiche. La cohésion sémantique, la vraisemblance fictive et la ressemblance avec l'hypotexte (si elles ne sont pas toutes la même chose au moins dans le premier cas) sont toutes maintenues en sa place.

Ayant pour but de mieux illustrer ce point nous avions attardé les exemples concernant les techniques de : "dissémination, condensation et déplacement". Il est désormais clair, espérons-le, le pourquoi de cette décision. Tous ces procédés sont manifestations différentes et spécifiques de cette attraction sémantique que nous avons appelée " aimants sémantiques". Nous allons donc emprunter les exemples, que l'on a souvent aussi constaté par voie directe, à Mercier-Leca. "Dissémination " : " le portrait du Prince de Conti sert-il à caractériser (...) et le duc de Montesquiou et le duc de Guiche ${ }^{79}$. Tous les deux personnages, amis de Proust et objets d'éloges dans le pastiche. "Condensation " : le portrait de Montesquiou est fait des emprunts, dont celui des " comtes de Bossu $»^{80}$ et celui que l'on vient de citer. L'auteur de ces exemples tire à cet égard un commentaire dont la connivence avec notre développement nous épargnera l'effort de toute explication : " Le portrait de Montesquiou est donc une rhapsodie, faite de fragments épars de portraits de Saint-Simon cousus ensemble $»^{81}$. Quant au "déplacement " enfin : on " attribue au duc d'Orléans une ignorance (...) des généalogies, que Saint-Simon impute à Louis XIV (... $)^{82}$ »

De la genèse littéraire nous allons aussitôt nous déplacer vers la dispositio et l'elocutio rhétoriques. Pour ce faire il nous faudra recenser la cohérence sémantique (telle que nous l'avons déjà amorcée) entre les manchettes et les parties du texte. On y a affaire à 8 manchettes, à savoir :

[I] Mariage de Talleyrand-Périgord. - [II] Succès remportés par les Impériaux devant Château-Thierry, fort médiocres. - [III] Le Moine, par la Mouchi, arrive au Régent. - [IV] Conversation que j'ai avec M. le duc d'Orléans à ce sujet. Il est résolu de porter l'affaire au duc de Guiche. [V] Chimères des Murât sur le rang de prince étranger. - [VI] Conversation du duc de Guiche avec M. le duc d'Orléans sur Le Moine, au parvulo donné à Saint-Cloud pour le roi d'Angleterre voyageant incognito en France. - [VII] Présence inouïe du comte de Fels à ce parvulo. - [VIII] Voyage en France d'un infant d'Espagne, très singulier ${ }^{83}$.

À partir de ces 8 manchettes, que nous allons désigner par des chiffres romains d'après leur ordre affiché, on constate qu'elles proposent 11 sujets. Pour arriver à cette somme on compte un sujet par manchette et puis on décompose la manchette III en deux sujets : conversation du pseudo Saint-Simon avec le Régent sur Le Moine et annonce de la décision (qui s'en découle) de parler à Guiche sur cela. À ce moment on en aura 9, il faut décomposer la manchette VI, partagée entre trois sujets : Conversation entre Guiche et le Régent, le parvulo à Saint-Cloud, la présence incognito du roi d'Angleterre en France. On doit y compter 11 sujets désormais.

Par des raisons d'espace, néanmoins, on ne va pas dégager ni traiter chaque thème quelle que soit la taille de son développement. Ce que l'on fera, par contre, 
sera essayer de saisir les phénomènes les plus importants qui s'y relient et qui relèvent de la cohérence sémantique. Évidemment on procédera par l'ordre de parution du texte, ou s'il en est le cas, par des conclusions générales tirées de notre lecture approfondie. Mais il y a encore un mot à dire, ou plutôt à répéter, sur les "intentions " sous-jacentes des déviations sémantiques. En résumé : les déviations relèvent soit de l'imitation du style saint-simonien, soit d'un portrait anachronique à fonction épidéictique-didactique. Cela nous confère une considération de plus pour apprécier la cohérence, cette fois-ci éthique, du texte, et pour préciser de quelle façon elle s'exprime. Le parangon continue à être la suite de manchettes, forme quasi paratextuelle qui nous est devenue fort utile.

Outre le fait que ces déviations sémantiques à caractère épidéictique y soient présentes, elles ne rompent ultérieurement pas la cohérence interne du texte, c'est dire elles ne font pas « dissonance ». En d'autres termes rappelons les mots de Barthes en parlant du récit:

On pourrait dire d'une autre manière que l'art ne connaît pas le bruit (au sens informationnel du mot) : c'est un système pur, il n'y a pas, il n'y a jamais d'unité perdue, si long, si lâche, si ténu que soit le fil qui la relie à l'un des niveaux de l'histoire. ${ }^{84}$

Certes, mais dans cette forme particulière d'art qu'est le pastiche l'unité esthétique de son système n'est pas gratuite, elle comporte l'exigence de conformité " ressemblance-différence " que les autres manifestions artistiques ne connaissent pas forcément. Dans ce sens l'art de portraire provient de ce qu'Aron nommait l'observation du social ${ }^{85}$, et l'imitation doit y céder le pas à la verve originelle de Proust, qui a en tête plusieurs portraits et commentaires de son milieu social. Pourtant il existe une assez grande " harmonie " entre les soucis des deux écrivains : Saint-Simon et Proust. Milly en témoigne : "Les préjugés nobiliaires et les questions de préséance, qui tiennent une place capitale dans les Mémoires, reçoivent dans le pastiche la même importance ${ }^{86}$.

Ayant ainsi établi l'équilibre interne du texte, et l'absence d'une menace causée par les déviations sémantiques, il ne nous reste qu'en soulever les exemples. Ces exemples sont parmi les nuances qui fournissent la forme finale du texte. Partons d'une conclusion générale : tous les portraits ou mentions, anachroniques autant les uns que les autres, qui adressent un blâme à un contemporain de Proust, figurent dans les manchettes ; et vice-versa : tous les éloges n'y sont pas annoncés ; à une exception près chacun, comme l'on le verra tout de suite.

Ceux-ci sont, en effet, les principaux personnages (contemporains à Proust) blâmés : Louis de Talleyrand-Périgord et Cécilia Blumenthal (mariage), le titre de duc de Montmorency qui avait M. Talleyrand-Périgord lui était contesté ${ }^{87}$, ensuite M. de Vendôme, contemporain de Saint-Simon ; certes son nom ne figure pas parmi les manchettes, mais il était engagé dans la campagne des Impériaux de la manchette II, et on le mentionne parce que la raison de son blâme (se faire appeler Altesse $)^{88}$ sert à introduire pour la première fois dans le pastiche : les Murat, déviation moindre mais dont l'importance va s'étaler par la suite ; son 
lien avec le texte est tout aussi clair alors. Probablement on pourrait proposer ici un autre avatar mécanique, dichotomique aussi, sur lequel repose l'équilibre, cette fois-ci sémantique du pastiche (dont le fonds dans ce cas est éthique) : " déviation-connexion " par rapport aux manchettes ; mais aussi au monde sémantique (et éthique!) saint-simonien, ce qui le relie avec l'équilibre paradigmatique du pastiche : "ressemblance-différence".

Ensuite, il y a les Murat, auxquels correspond la manchette V et de pair le blâme le plus long et le plus sévère du pastiche. Il s'agit, en effet, d'une famille de noblesse étrangère (Naples) qui dérange la noblesse parisienne par ses prétentions ${ }^{89}$. La présence de J. Murat au parvulo de Saint-Cloud sert de pont pour introduire les sujets de la manchette VI. À l'intérieur de la manchette VI on retrouve la véritable exception à la norme proposée, il s'agit du mariage du duc de Cambacérès, dont on a déjà quelque peu parlé. Or il faut noter qu'il présente une autre particularité concernant son caractère épidéictique : les personnages qui s'y marient ne sont pas contemporains de Proust, ni de Saint-Simon non plus, ils appartenaient à la noblesse d'Empire, celui qui est contemporain de Proust et qui est attaqué pour prendre le titre de comte de Cambacérès, est Maurice Delaire ${ }^{90}$. Quant à la manchette VII, c'est bien déterminé qu'il s'agit de censurer le comte de Fels (comte Edmond Frisch de Fels) ${ }^{91}$. Enfin pour ce qui est de la manchette VIII l'infant d'Espagne n'est autre que Louis-Ferdinand d'Orléans qui effectivement portait ce titre ${ }^{92}$, et qui faisait l'occasion d'un malaise de l'aristocratie contemporaine de Proust.

Or notamment dans les parties consacrées au développement des reproches aux Murat et à l'infant d'Espagne, Proust emprunte divers passages " analogues " (ou susceptibles de le devenir) des Mémoires, pour rendre son blâme dans la langue de Saint-Simon, ainsi qu'il a fait avec les éloges et avec les portraits en somme. Ceci a été bien établi depuis l'édition critique des pastiches de Proust par Jean Milly, à laquelle nous renvoyons pour retrouver les précisions dues à ces emprunts. Ce qui nous intéresse, c'est le fait de relever à cet égard, de nouvelles preuves, s'il en était besoin, du procédé de convocation sémantique, que nous avons appelé " aimants sémantiques ". Sauf que dans ce cas il serait géré par un raisonnement analogique, c'est-à-dire métaphorique, mais dont la métaphore reste du côté de la conception, soit de la création pure, et ne figure pas dans le texte comme telle.

Passons donc, tout de suite, à analyser le cas des éloges dans le pastiche. Le tout premier éloge, est tout de même l'exception à la règle : il apparaît dans les manchettes. C'est le cas de Guiche dans la manchette IV, or cette mention recouvre aussi une particularité assez spéciale : le duc de Guiche (celui des Mémoires) est le véritable ancêtre de Guiche l'ami de Proust qui se voit largement flatté dans le pastiche, et parmi les personnages qui s'y voient loués, c'est lui, qui par le nom peut-être le plus approché des scènes engageant aussi le « pseudo Saint-Simon » et le Régent, ce qui est son rôle dans la fiction ; en plus de maintenir de ce fait la ressemblance entre l'hypertexte et son hypotexte. Dans la machette $\mathrm{V}$ alors qu'il est question du parvulo, c'est la partie du texte où il y a le plus de louanges comprises. D'abord c'est le tour de la princesse Soutzo, amie de Proust, 
qui s'y voit introduite par la conversation entre le " pseudo Saint-Simon " et les ducs de Mortemart et de Chevreuse, ceux-ci rapportant une " réponse qu'elle avait rendue $»^{93}$ à propos des "prétentions de J. Murat".

Deux longs paragraphes après, c'est le mariage Asquith-Bibesco ${ }^{94}$ qui est loué, surtout grâce à un effet de contraste avec le mariage Delaire-Rohan qui figure juste avant. Le couple Asquith-Bibesco est aussi parmi les amis de Proust. On y introduit une petite mention de Paul Morand (ami de Proust), et voici ce qu'on dit : " duquel il sera souvent question au cours de ces Mémoires $»^{95}$ on verra que Proust considérait l'idée de composer une continuation du pastiche de SaintSimon. Puis il est question de Robert Montesquiou-Fézensac, dont nous avons déjà assez parlé. Mais il y a d'autres portraits qui se trouvent là-dedans, et dont nous allons parler, mis à part, et pour cause, celui d'Yturri. Ainsi en parlant du cercle social de Montesquiou, on mentionne : Mme Greffulhe (cousine de Montesquiou) ${ }^{96}$, Mme de Noailles (poétesse ami de Proust, et véritable descendante des Noailles des Mémoires), Mme Straus (ami de Proust et souteneuse d'un salon dont il était très assidu) ${ }^{97}$, Mme Standish (cousine aussi de Montesquiou, présente au parvulo, et objet d'un éloge paradoxal tant on se moque de sa prétention de ressembler à la princesse de Galles ${ }^{98}$, et qui dans le pastiche se reflète par le commentaire que le Régent tient à propos de ses perles qu'elle portait comme la reine d'Angleterre ${ }^{99}$ ).

De " retour " au parvulo, on mentionne : milord Derby, B. Lytton et milord Dufferin, tous les trois diplomates ou ambassadeurs anglais du temps de Proust ${ }^{100}$, que l'on rapproche à l'ancienne cour anglaise dans le pastiche. Après, il y a Olivier (maître d'hôtel du Ritz) ${ }^{101}$. Finalement dans ce qui correspond à la manchette VII, nous retrouvons le seul éloge des comtes de Beaumont (dont les noms sont présents dans les Mémoires, mais qui dans ce cas il s'agit des amis à Proust) ${ }^{102}$.

En d'autres termes, nous pouvons relier ces portraits à deux caractéristiques essentielles de l'œuvre saint-simonienne, relevant chacune de domaines différents, auxquels nous retournons : l'éthique et la sémantique. Nul n'ignore que tout l'univers social saint-simonien est coupé en deux morceaux par un principe de moral : " les honnêtes gens " et "le reste du monde qui était en faute par quelque raison qui ce fût ". Cela se reflète dans le texte par une narratio épidéictique de quelque côté qu'elle se penche. Dans ce sens, telles peuvent être la fonction et l'intention principales des Mémoires.

Puis cette même " obsession" (qui était connue à la cour, même par le roi) ${ }^{103}$ est à l'origine des longues déviations du sujet du texte. Elles créent un effet de suspense dont Proust a su aussi tirer large profit dans son imitation. En fait, lorsqu'on lit le pastiche pour la toute première fois et que l'on s'attend à ce que la thématique majeure soit L'Affaire Le Moine on est sujet d'un continuel suspense, qui s'opère, d'après Barthes : " par des procédés emphatiques de retard et de relance $»^{104}$. Ce suspense connaît dans le texte une péroraison pathétique de chute ou par'hyponoian, tant les attentes sont trompées, car ladite affaire n'est qu'une excuse littéraire et qu'elle n'est même pas résolue dans le pastiche. Cette démarche : longue protase - courte apodose, s'agençait aussi par des excuses que le narrateur offre dans ce sens : "L'affaire du Moine ne touchait pas à des inté- 
rêts si vitaux pour la France ${ }^{105}$ ou encore la fin du texte : " Mais cette disgression sur les titres singuliers nous a entraînés trop loin de l'affaire du Moine ${ }^{106}$. Excuse, effet comique, mais aussi justification pour rédiger une suite.

\section{F. La voix narrative hypertextuelle}

Tous les paniers géants, les justaucorps à basques, Dans ce double cristal, vont se multiplier ; Et des perles en pleur, des larmes en collier Roulent au bord des yeux, lorsque tombent les masques ${ }^{107}$

Certes, nous proposons ici, jusqu'à notre connaissance, une terminologie nouvelle qui dérive manifestement d'un hybridisme entre la narratologie et l'hypertextualité. Nous en avons déjà dûment signalé les sources théoriques. Il s'agit maintenant d'amorcer cette notion à partir des interrogations qui nous ont poussés dans cette direction. Ce qui nous intéresse par-dessus tout sont les enjeux de l'identité du narrateur, et les masques qu'il doit porter dans ce spectacle si particulier du pastiche. On parle bien de spectacle parce qu'il nous intéresse de relever tantôt les faits de la création, tantôt ceux de la réception, et que l'on va considérer tout ce phénomène comme un spectacle artistique qui englobe des faits extérieurs au texte que nous préciserons en son moment, et qui tous ensemble comporteront un " corps esthétique " à être analysé. Ce " corps esthétique" sera par moments objet d'une analogie métaphorique avec le théâtre dont nous allons emprunter quelque vocabulaire, pour pouvoir donner, espérons-le, une finalité heuristique à ce rapprochement métaphorique.

Or, toute la discussion sur ce " corps esthétique » sera orientée vers le but de préciser les enjeux narratifs qui relèvent surtout de la voix dans la narration. On laissera de côté les aspects plus propres à la structure interne du récit, et l'on cherchera préférablement de mesurer les implications extérieures (esthétiques et éthiques) auxquelles est soumis l'auteur-narrateur dans son rôle de créateur, de metteur en scène et de personnage de la fiction même qu'il a conçue. Malgré l'extériorité de beaucoup d'éléments composant l'échafaudage de ce « corps esthétique ", on verra par la suite, que tous ces éléments sont en fait des conditions requises dans la mise au point du pastiche de Saint-Simon ; ils reviennent donc dans une certaine mesure à l'établissement de la cohérence interne du texte. Ce que d'une façon tout à fait réductrice on pourrait décrire, en affirmant que si ces éléments extérieurs avaient été différents (et cela est mesurable), on aurait affaire à un texte tout autre.

C'est par ces raisons que l'on a décidé de nommer cette réflexion (faute de mieux, peut-être) : " voix narrative hypertextuelle ", en considérant que la plupart des conditions qui doit accomplir l'auteur-narrateur peuvent s'identifier (quelque peu tout au moins) avec les faits narratifs de la voix, et que la plupart des spécificités et presque toute la subtilité de son art peuvent être attribuées à la nature hypertextuelle du pastiche. On suppose qu'en principe les pastiches 
hypothétiquement parlant relèvent, comme nous l'avons marqué, d'un mimétisme stylistique, bien entendu, la narration y devrait être comprise. Or, stylistique et narratologie, sont deux démarches de critique littéraire différentes, mais lorsqu'on met en place une imitation d'un récit narratif, cette imitation comprend, et pour cause, la narration. Ce qui octroie le rang de particularité à ce pastiche est tout le spectacle monté autour des messages qui appartiennent exclusivement à l'imitateur et au fait, qu'ils doivent, comme nous l'avons signalé, s'accorder au modèle et, comme nous le verrons, "s'expliquer " en raison du public auquel ils sont adressés.

Quoiqu'il en soit, il reste encore quelques questions de base, c'est-à-dire antérieures à la réception du texte, et qui méritent notre considération explicite. Elles consistent notamment à se demander une description en thermes esthétiques, et probablement philosophiques, de la tâche du narrateur pasticheur. Songeons désormais à tenir en compte cette réflexion de Genette à propos de l'acte narratif, et de l'énorme importance de sa nature fictive :

Si (...) on tient Ulysse pour un menteur, et pour fictive les aventures qu'il raconte, l'importance de l'acte narratif ne fait que s'en accroître, puisque de lui dépendent non seulement l'existence du discours, mais la fiction d'existence des actions qu'il « rapporte ${ }^{108}$.

Maints raisonnements peuvent être tirés de cette citation si précieuse, voyons ce que nous saurons en dégager. Le pastiche est évidemment un acte fictif, comme l'est par exemple un roman. La narration suppose alors que l'auteur laisse de côté sa personnalité et assume quelque autre, que ce soit narré à la première ou à la troisième personne, peu importe. En ce sens l'auteur est un acteur dont le personnage est le narrateur. Son art consiste donc à adopter une perspective étrangère, dans le cas du roman ou du conte, elle est le plus souvent inventée, sinon est-elle supposée (assumée), lorsqu'elle retrouve quelque référent réel d'inspiration. Dans le cas du pastiche, ce personnage (celui qui doit adopter le narrateur) est quelqu'un de réel, et qui plus est, quelqu'un qui par le moyen de ses œuvres a laissé toute une " didascalie " qui sert à ce que l'on interprète son personnage (le narrateur dans ses œuvres) à bon escient. Or, cette " didascalie » est encryptée, et l'auteur du pastiche est obligé d'en connaître d'abord le code pour pouvoir devenir narrateur, soit pour pouvoir interpréter ce personnage.

En tout cas, quelle que soit le genre (roman ou pastiche), l'apparence esthétique du récit narratif dépend et de l'identité et de la perspective engagées par l'artiste. Plus le rôle est spécifique, plus l'esthétique du texte est prédéterminée. Cela est vrai dans ce pastiche, car l'intention de Proust n'y est point caricaturer le style de Saint-Simon ; le duc ni son style n'y sont pas la cible. En fait, la cible en est le public qui s'y voit inclus, et cela entraîne que Proust se consacre largement à saisir ce " personnage ", puisque cela donnerait une portée beaucoup plus transcendantale à ses messages didactiques-épidéictiques. Il y est question de " s'habiller » du costume solennel du grand juge de la haute société française et de recréer une illusion telle à ce que ce public visé ait, par instants tout au 
moins, l'impression que ce grand monsieur parle d'eux. On va, en amont, remprunter à Proust même ce passage de la Recherche avec l'ajout de Luc Fraisse, et dire qu'il s'agit de « voir et donner à "voir l'univers avec les yeux d'un autre» » ${ }^{109}$.

C'est ainsi que la narration, selon cette perspective est toujours un acte d'imitation, de "mimésis". Genette, ramasse à ce titre, l'ancienne définition de Platon sur la narration : " «[s'] il s'efforce de donner l'illusion que ce n'est pas lui qui parle», mais un tel personnage ${ }^{110}$. Cela vient renforcer notre position du début, selon laquelle la différence entre le pastiche et tout autre acte narratif fictif, est la nature de ce "personnage». Encore comme dans le bricolage et dans la critique littéraire, dans le pastiche, l'auteur se voit forcé de composer son masque de narrateur à partir des matériaux fournis par l'hypotexte, tandis que le romancier dispose de "tout l'univers » pour ce même travail.

Mais il ne faut pas trop s'avancer dans ce sens, en oubliant ce que l'on a déjà établi, à savoir que cette imitation doit être reconnue, ce qui n'est pas du tout le cas du roman. C'est donc pour cette cause que selon Genette l'état idéal du pastiche est l'imitation perceptible comme telle ${ }^{111}$. Nous répétons à nouveau cette condition sauf que cette fois-ci ce qui nous intéresse n'est pas exclusivement sa lecture mais le rôle du narrateur à cet égard. Proust dirigeant ainsi certains messages à un public, il doit accomplir l'illusion suffisante de ressemblance au modèle, mais il faut simultanément que l'illusion ne soit pas " absorbée " complètement par le public, pour ainsi dire. C'est le cas le plus extrême et le plus explicite de ce que Barthes décrivait comme : " «larvatus prodeo», je m'avance en désignant mon masque du doigt ${ }^{112}$. Ce masque enfin est celui du pasticheur : moitié Proust imitant (identifié avec l'auteur, en raison des messages que Proust envoie à son propre cercle social), moitié pseudo Saint-Simon imité.

Ce rôle paradoxal peut mieux s'illustrer par ce que l'on dit de Robert Montesquiou dans le pastiche : "il riait fort de ce qu'il disait comme s'il avait été à la fois l'auteur et le parleur ${ }^{113}$. Il faut y faire très attention au choix de mots, c'est une dichotomie par'hyponoian, on s'attendrait à ce qu'il dise plutôt auteur-récepteur. Cela est susceptible d'indiquer que pour Proust, l'autorité est un acte restreint à la conception et que l'expression peut se faire à travers quelqu'un d'autre (un personnage), ou l'auteur même adoptant une autre personnalité, ce qui n'est autre chose que le narrateur. C'est comme dans le théâtre où il est possible que l'acteur accomplisse la tâche de créateur, c'est-à-dire de dramaturge, mais il faut toujours les séparer, puisqu'elles relèvent de niveaux différents, tout comme le personnage qui est à la fin de la chaîne.

Or, on doit convoquer certaines particularités du modèle qui déterminent profondément la forme narrative du pastiche. Selon Genette, la mimésis (que non le mimétisme !) se définit " par un maximum d’information et un minimum d'informateur ${ }^{114}$. Mais est-ce le cas du pastiche en question ? Il ne l'est certes pas, parce que nous y avons affaire à un mimétisme d'une diégésis, car on considère les Mémoires un récit diégétique à part entière. Cela est ainsi puisque le texte de Saint-Simon fonctionne au rebours du schéma de la mimésis, qui figure en haut. Une des principales caractéristiques de ce fait est la prééminence de la "fonction idéologique ", que Genette définit, à peu près, comme les interventions, 
directes ou indirectes, du narrateur à l'égard de l'histoire, ayant la forme d'un commentaire didactique ${ }^{115}$; ce qui est précisément le cas des Mémoires.

On n'a pourtant pas l'intention de trop approfondir dans l'aspect narratif des Mémoires, mais on songe à soulever les éléments les plus importants de la voix narrative, qui déterminent l'enjeu esthétique de la narration dans le pastiche. On connaît tous de nos jours la distinction entre les entités d'auteur et de narrateur, qui est absolument claire, d'ailleurs, dans presque tous les genres littéraires, hormis dans un récit historique ou autobiographique ${ }^{116}$. En conséquence, on peut dire que Saint-Simon est tantôt auteur, tantôt narrateur de son récit et que la seule différence se trouve au niveau du narrateur et elle est de type temporel : " je narrant " et " je narré ${ }^{117}$. Ce décalage a été poétiquement saisi par Jean-Michel Delacomptée :

L'audace de se lancer, à soixante-cinq ans, sans espoir ni volonté d'être publié de son vivant, isolé dans son cabinet avec l'ombre des morts pour compagnons de voyage, dans une odyssée égale par la durée, sinon par les périls, à celle du roi d'Ithaque de retour vers son île de même que, muni de sa plume en guise de nef, Saint-Simon s'en revint vers l'archipel de la cour ${ }^{118}$.

Proust conscient de cette réalité, doit assumer l'imitation d'un auteur-narrateur presque insécable, cela décerne plus de "réalité ", pour ainsi dire, au référent imité et amenuise, par là même, l'effet d'illusion esthétique chez le lecteur. L'une des toutes premières prémisses de notre travail est la différence irréductible dans l'expérience esthétique lors de la lecture d'un roman, par exemple, et le pastiche de Saint-Simon par Proust. La lecture d'un roman pourvoit une illusion plus forte et plus régulière de l'identité de son narrateur, qu'il soit héros ou non du roman. Ce que nous ramassions tout à l'heure sur l'importance de la fiction dans la narration, l'auteur n'y est contraint à " aucune " conformité pour constituer l'identité de son narrateur, il est libre de choisir l'apparence esthétique qui lui semble convenable. Au contraire, dans ce pastiche Proust n'est pas seulement contraint par l'acte de l'imitation sinon aussi par la tangibilité narrative du modèle choisi.

Cela dit, ce que nous envisageons d'en repérer est le fait que l'expérience esthétique de la lecture est bien plus complexe que la complète illusion du roman. Puisque, tout au long de la lecture du pastiche, on est censé apprécier la beauté du texte qui consiste en la réussite de cette forme "d'imitation hommage " du style saint-simonien et simultanément dans la "fausseté " de l'identité du narrateur : le pseudo Saint-Simon. C'est donc un spectacle sophistiqué de tout point de vue, Proust aurait pu diriger et ses politesses et ses reproches d'une autre façon, plus simple peut-être, mais il a choisi celle-ci. C'est en ce sens que le spectacle du pastiche de Saint-Simon est proche du théâtre : tout le monde regarde le pseudo Saint-Simon en scène en portant son masque, son costume, et cela implique, et pour cause, que ce monde aperçoit aussi, tout en même temps, Proust qui en joue le rôle.

En plus, si nous avons déjà signalé la " vocation épidéictique » du pastiche, et le choix exprès de Proust de rendre ce message sous cette présentation (il ne 
faut pas oublier que "fête chez Montesquiou à Neuilly" a été publié en 1904)"119, il nous faudrait encore repérer le pourquoi. L’une des particularités du pastiche est que les personnalités qui y font l'objet des blâmes ou des éloges sont mentionnées directement ou presque. Nous avons remarqué, en plus, la portée que ces discours épidéictiques auraient étant habillés de la voix du duc de Saint-Simon. Mais il nous faut désormais remarquer (et voilà le pourquoi du choix) l'oblicité esthétique du message. Nouveau paradoxe, bien entendu, à l'intérieur de notre analyse, car cette technique a été pratiquée depuis l'antiquité, les fables en témoignent ou sinon moins éloigné dans le panorama de la littérature française, pendant le XVIIIe siècle maints exemples pourraient être ajoutés, qu'il nous suffisse d'en citer pour l'occasion Les lettres persanes de Montesquieu.

On a bien compris depuis, que le plus oblique est la perspective narrative du message, le plus hardi peut être ce message, et qu'il sera toujours (variablement) accueilli chez le public. Pourtant l'oblicité du pastiche L'Affaire Le Moine ne réside pas exclusivement dans la perspective de l'instance narrative choisie, sinon que tout le milieu est rapporté de façon oblique par la voie d'une transposition analogue entre sociétés et ses intégrants. C'est-à-dire qu'hypothétiquement parlant Proust aurait pu supposer ce que Saint-Simon dirait s'il arrivait et qu'il voyait son cercle social. Mais l'expérience est menée au rebours, Proust se transpose, et avec lui sa petite société, à la cour du Régent. Ce fait est à l'origine de ce que nous affirmons que Proust n'est pas seul « sur scène ", son cercle social l'accompagne (on reviendra sur ce fait), et cette licence artistique est atteinte grâce à l'oblicité du milieu du récit tout entier, y compris la perspective narrative. Ce milieu fictif évidemment, est un " décor " qui flatte l'esprit de certains gens, surtout de ceux qui s'y voient loués, car bien que fiction elle évoque une notion qui est conçue comme une réalité pour ce public: effet pathétique.

Ainsi, tout comme la syntaxe de la phrase proustienne, le pastiche proustien est un jeu des renvois constants et multipliés, cette fois-ci entre la "réalité " et la " fiction ». Le spectateur, notamment celui qui fait partie de la petite cible et par conséquent de ce que l'on a nommé ici le " corps esthétique ", est sujet d'une houle de stimuli esthétiques et éthiques qui requièrent de plusieurs degrés d'observation, tant chaque stimulus présente ce paradoxe d'équilibre : « ressemblance-différence " dont nous parlions abondamment ailleurs.

La relation création-réception est si étroite dans ce texte que Proust en s'en apercevant a écrit ces deux morceaux sagement rattachés par Luc Fraisse :

Les pastiches, (...), supposent un public choisi et de choix (...) « ces pastiches, (...) explique Proust (...) sont en effet de petits exercices pour lesquels il n'y a pas besoin de talent, mais qui ne s'adressent qu'aux gens de talent [...] parce que seuls ils peuvent comprendre la plaisanterie du pasticheur! $»^{120}$

Est-ce la cause de ce qu'aucune des personnalités louangées, à une exception près, ne figurent pas parmi les manchettes ? Est-ce pour que les "gens de talent " auxquels se dirige le pastiche, en plus que flattés se sentent eux surpris par cette courtoisie? Et que les autres (les blâmés) craignent juste en voyant les 
manchettes ? Tout pourrait être. Ce qui est particulier dans cette situation, est le fait d'être capable d'utiliser une forme littéraire ancienne et d'en remporter les mêmes résultats, cela veut dire que Proust a eu du succès pour rencontrer une petite société analogue à celle de Saint-Simon, et par là même, maintenir la fonction " originelle » de cette forme littéraire. Ce qui ne va pas de soi, comme en témoigne ce raisonnement de Genette :

Chaque œuvre se trouve orientée par rapport au milieu littéraire, et chaque élément par rapport à l'œuvre entière. Tel élément qui a sa valeur déterminée à une certaine époque changera complètement de fonction à une autre époque ${ }^{121}$.

Encore dans cette étroite relation de création-réception, il y a un fait qui pèse énormément et qu'il nous reste à analyser. Ce fait se reflète dans les relations de Proust avec son cercle social, principalement avec Mme Straus et Robert Montesquiou-Fézensac. Ainsi Proust, lorsqu'il se consacre à la mise au point du pastiche, il envoie quelques lettres notamment à Mme Straus ${ }^{122}$ et à la princesse Soutzo $^{123}$ pour leur faire part de leurs apparitions dans le pastiche, et des autres gens qui y auront son lieu. Spécialement dans la correspondance à Mme Straus, il est question de l'importance de la mention médisante des Murat. En retournant sur notre métaphore entre le " corps esthétique " du spectacle du pastiche et le théâtre, on trouve que ces lettres qui relèvent de la paratextualité ont son " équivalent " dans le théâtre. Au fait, et à condition d'y percevoir la création et la réception du pastiche comme un même spectacle, le théâtre classique disposait d'une technique " analogue " qui n'est guère utilisée de nos jours : la parabase.

Outre, l'heureuse coïncidence des préfixes, on se rend compte que la technique de l'ancienne comédie classique, a une nature en commun avec la paratextualité dans le cadre stricte de ce cas. Certes la nature des arts sépare temporellement le lieu où l'on peut se servir de ces techniques, c'est par cette raison entre autres, que l'on décide d'élargir le champ d'observation esthétique du spectacle du pastiche. Mis à part, ce décalage d'essence temporelle, toutes les deux ressources (il vaut mieux d'ainsi les nommer) supposent une sorte de pause dans le spectacle, la rupture de l'ingénuité du spectateur, un clin d'œil, enfin, de la complicité et de l'intelligence, qui a besoin d'un public capable de le recevoir. Proust élabore donc son spectacle par étapes, il y mène ses expériences pour " tâter le pavé " ${ }^{124}$ et il en obtient des résultats : "Pastiches et Mélanges se lira d'autant plus que toute une partie de la société parisienne paraît dans le Pastiche de Saint Simon " ${ }^{125}$.

Or, la relation Proust-Montesquiou est beaucoup plus intéressante pour nos propos. Comme nous l'avons préalablement repéré, Robert Montesquiou est l'objet du principal éloge du pastiche. En fait, son inclusion dans le pastiche est à l'origine d'un jeu littéraire entre les deux écrivains. En 1899, Montesquiou a publié son recueil de poésie : Les perles rouges ${ }^{126}$ dont le sonnet XLIII est un hommage à Saint-Simon, on l'y rapporte :

L'œil était dans la tombe...

Le vrai Louis Quatorze est le seul Saint-Simon, 
Le Grand Siècle écoulé survit en son grimoire :

Tapisserie énorme, inexorable moire

Qu'ourdit une Arachné moins ange que démon

Louis mène le char. Le Duc est au timon.

Les rayons du Soleil rentrent dans son armoire.

Les Dieux ne seront pas grands que selon sa mémoire.

L'astre n'aura d'orgueil que selon son gnomon.

Nul ne sait qu’il écrit, ce Mémorialiste!

Des brebis de des boucs il dresse une liste,

Et sa lampe nocturne est un phare immortel.

Il note, à leur insu, Beauvilliers, Albermarle,

Celui-ci, d'un gibet, celui-là d'un autel...

Puis, quand le mort est bien au fond de l'ombre... IL PARLE !127

C'est donc à partir de ce texte, selon le rapporte Milly, que Proust conçoit le projet de lui " rendre la politesse " ${ }^{128}$ et qu'il rédige " Fête chez Montesquiou à Neuilly " qui apparaît dans Le Figaro du 18 janvier 1904, sous le pseudonyme d'Horatio, une fois que Montesquiou se réjouissait du texte, Proust en a admis l'autorité ${ }^{129}$. Puis Montesquiou est présent tout de même, et en occupant la place que nous avons dit, dans le pastiche. Il s'agit d'un jeu littéraire de société, d'un divertissement artistique. On est peut-être à la source d'une des principales raisons pour lesquelles Proust décide de porter le masque du pseudo Saint-Simon, il veut offrir " une réponse " à Montesquiou, plus qu'une politesse c'est une courtoisie flatteuse, un régal d'une sophistication extrême.

Proust, " connaissant " a priori, le succès de son pastiche, envisageait d'en rédiger une suite. Cette caractéristique est partagée de son autre hypertexte composé préalablement : "Fête chez Montesquiou à Neuilly ", mais aussi fort curieusement du texte source : Saint-Simon comptait, à cet égard, sur la continuation de ses Mémoires. On souhaite avec zèle, quant à nous, avoir l'honneur de rejoindre ce bel esprit d'espoir de poursuite, en cherchant à se faire entendre quand on en aura, encore, quelque chose à dire.

\section{Conclusions}

Cette étude du pastiche Le Moine a supposé une mise en preuve des certaines prémisses interprétatives et méthodologiques, qui ont été suffisamment expliquées dans leurs compartiments respectifs. On considère désormais, ayant mené l'exercice jusqu'au bout, que l'on a, tout au moins, quelque peu contribué dans le champ de la discussion théorique de ce pastiche, peut-être surtout, telle était notre intention générale, dans l'éclaircissement des enjeux éthiques et esthétiques qui s'opèrent tantôt à son intérieur, tantôt autour de lui. Tout de même 
que la signalisation particulière du lien existant entre ces deux pôles (intérieurextérieur du texte), dans le cas de ce pastiche. Ces " contributions " on peut les résumer en trois grands axes, distinguées par leur direction théorique, que l'on va noter par gradation, selon le degré d'innovation que l'on y entend retrouver.

De prime abord, on s'est fait répercussion des échos divers de la particularité stylistique et esthétique de l'activité pastichante de Marcel Proust, et l'on suppose avoir continué, à bon escient, cette tradition, surtout par ce qui concerne le pastiche de Saint-Simon et sa singularité, même vis-à-vis des autres pastiches de Proust. On entend, compte tenu de la gigantesque taille de l'œuvre proustienne, avoir soulevé, grâce à la rigueur de notre démarche, certaines réflexions probablement utiles pour la suite des études proustiennes. Notamment, il nous semble d'une valeur fondamentale l'établissement de l'équilibre esthétique de l'art du pastiche, que l'on a nommé par la dichotomie : " ressemblance-différence".

Ensuite, on pense avoir soulevé une perspective d'analyse intéressante et d'une estimable valeur heuristique, par la voie de notre proposition de la " cohérence sémantique ». Principalement par le fait que cette perspective a permis de joindre, en grandes lignes, la sémantique avec l'éthique, et par là même : l'intérieur et l'extérieur du texte pour ainsi dire. Cela a comporté, dans une certaine mesure, une évocation de l'ancienne rhétorique : "dispositio-elocutio " et ethos aussi, mais il faut remarquer que cette évocation s'est faite (paradoxalement ?) par le biais d'une démarche plutôt structuraliste. Ainsi, on a vu figurer côté à côté, et de façon cohérente, des réflexions portant sur des considérations créatrices, dont principalement celle des " aimants sémantiques » et d'autres portant sur l'intention et la réception du texte, comme lorsqu'il est question de la vocation épidéictique-didactique du pastiche.

En fin de comptes, on croit assurément avoir réussi à élargir quelque peu le champ typique de réflexion de ce pastiche, en y rapprochant la narratologie, et quelques notions théâtrales de nature analogue, du moins pour les effets de cette analyse. On estime avoir pu générer, par cette manœuvre, un objet d'étude plus complet et plus révélateur : le " corps esthétique " qui suppose un pas de la considération plus restreinte du pastiche comme stricte lecture à l'agencement équilibré des donnés pour le regarder d'un mode plus large : celui d'un spectacle, dans toute sa magnificence esthétique. Dans ce même sens, on considère avoir suffisamment amorcé les implications identitaires dans la tâche du narrateurpasticheur et de son étroit rapport avec le milieu récepteur qui en détermine certaines conditions essentielles. Un phénomène que selon nous est l'épicentre de la constitution de l'expérience esthétique du pastiche L'Affaire Le Moine, puisque tel que l'on l'a vu, il est juste à mi-chemin entre la création et la réception.

\section{Notes}

1 Proust, Contre Sainte-Beuve, éd Pierre Clarac et Yves Sandre, Bibliothèque de la Pléiade, 1971, p. 25. 
2 Proust, Les Pastiches de Proust, édition critique et commentée par Jean Milly, Armand Colin, 1970, p. 20.

3 Voir. Proust, op. cit., éd. Jean Milly, p.292. Jeu tiré des variations saint-simoniennes sur Le Charmel/Charmel.

4 Gérard Genette, Palimpsestes, Éditions du Seuil, coll. Points, 1982, p.10.

5 Voir. Gérard Genette, Figures I, Éditions du Seuil, coll. Points, 1966, p. 43. Le « Vernis des Maîtres ».

6 T. Todorov, Théorie de la littérature, Éditions du Seuil, 1965, p. 37.

7 On garde cette graphie et non la forme plus commune "épidictique », par des raisons d'étymologie, cette forme étant plus proche du mot « deixis » (mot qui deviendra significatif à un moment donné de ce travail), toutes les deux portant le sens de montrer quelque chose. Ainsi : gr. $\varepsilon^{\prime} \pi 1 \delta \varepsilon 1 \kappa \tau 1 \kappa o^{\prime} \varsigma$ - lat. epidicticus : « qui sert à montrer ».

8 Paul Aron et Florence Mercier-Leca.

9 Voir. Proust, op. cit., éd. Jean Milly

10 Ibid. p. 238.

11 Gérard Genette, Palimpsestes, op. cit., p. 13.

12 Voir. Gérard Genette, Figures III, Éditions du Seuil, coll. Poétique, 1972.

13 Ibid. p. 202.

14 Gérard Genette, Palimpsestes, op. cit., p. 8.

15 Ibid.p.8

16 Bille Jørgensen Steen, «Pastiche et poétique de l'œuvre. Stratégies de réécriture contemporaines ", Revue d'histoire littéraire de la France, 2012/1 Vol. 112, p. 114.

17 Stéphane Caillé, Proust et la recherche des répétitions, le cas de l'intertextualité, Thèse de Doctorat, Université de Montréal, 1999.

18 Dominique Jullien : Proust et ses modèles, "Les Mille et Une Nuits » et les « Mémoires » de Saint-Simon, José Corti, 1989.

19 Gérard Genette, Palimpsestes, op. cit., p. 43.

20 Ibid. p. 45.

21 Ibid. p. 106.

22 Proust, op. cit., éd. Jean Milly, p. 25.

23 Gérard Genette. Figures III, op. cit., p. 13

24 Florence Mercier-Leca, «Saint-Simon au miroir : trois pastiches des Mémoires aux XXe et XXle siècles », Littératures classiques, 2011/1 № 74, p. 211.

25 Luc Fraisse, «Proust : philosophie du pastiche et pastiche de la philosophie », Revue d'histoire littéraire de la France, 2012/1 Vol. 112, p. 71.

26 Mercier-Leca Florence, art. cit.

27 Paul Aron, «Les pastiches littéraires dans À la recherche du temps perdu », Revue d'histoire littéraire de la France, 2012/1 Vol. 112, p. 53.

28 Ibid. p. 6.

29 Boileau, L'Art Poétique, Garnier, p. 2, v. 37-38.

30 Gérard Genette, Figures III, op. cit., p. 73.

31 Gérard Genette, Palimpsestes, op. cit., p. 117.

32 Bille Jørgensen Steen, art. cit. p. 105. « Le pastiche plus qu'un genre est une pratique qui explore les limites du roman (ou du genre imité, dirions-nous). »

33 Gérard Genette, Palimpsestes, op. cit., p.104.

34 Paul Aron, «Sur les pastiches de Proust l'ethos et le champ » sur le site revues.org/ contextes, p. 1.

35 Proust, op. cit., éd. Jean Milly, p. 25. 
36 Cf. Proust, op. cit., éd. Jean Milly, p. 26.: « La fonction de référence se combine avec la fonction métalinguistique. 》

37 Ibid. p. 29.

38 Paul Aron, «Sur les pastiches de Proust l'ethos et le champ », art. cit., p. 6.

39 Gérard Genette, Figures I, op. cit., p. 145.

40 Voir. Proust, op. cit., éd. Jean Milly, p. 29.

41 Voir. Gérard Genette, Palimpsestes, op. cit., p. 31.

42 Roland Barthes. Introduction à l'analyse structurale des récits. In: Communications, 8/1966. Recherches sémiologiques : l'analyse structurale du récit. p. 26.

Proust, op. cit., éd. Jean Milly, p. 18.

44 Gérard Genette, Palimpsestes, op. cit., p.104 sqq.

45 Florence Mercier-Leca, art. cit., p. 203.

46 Gérard Genette, Palimpsestes, op. cit., p.140.

47 Avant-propos à la Comédie Humaine.

48 Proust, op. cit., éd. Jean Milly, p.29. Note 10 en bas de page.

49 Gérard Genette, Palimpsestes, op. cit., p.102.

50 Voir. Gérard Genette, Palimpsestes, op. cit., p.135. « Le pastiche-hommage ».

51 Proust, op. cit., éd. Jean Milly, p. 289.

52 Gérard Genette, Palimpsestes, op. cit., p.159.

53 Gérard Genette, Figures I, op. cit., p. 56.

54 Proust, op. cit., éd. Jean Milly, p.29 sqq.

55 Luc Fraisse, art. cit., p. 66.

56 Saint-Simon, Mémoires, éd. Yves Coirault, Gallimard, coll. Folio, 1990, p. 307.

$57 \quad$ Ibid. p. 573.

58 Voir. Proust, op. cit., éd. Jean Milly, p. 310.

59 Florence Mercier-Leca, art. cit.. p. 204.

60 Gérard Genette, Palimpsestes, op. cit., p. 115.

61 Voir. Proust, op. cit., éd. Jean Milly, p. 35.

62 D. C. Cabeen, «Proust and Saint-Simon », Modern Language Association, Vol. 46, No. 2, 6/1931, p. 608-609 : «Proust soulève là, parfois même de façon burlesque, les qualités plus que connues du Duc : son insatiable curiosité pour le commérage anecdotique, sa vanité de grand seigneur, sa haine contre la bourgeoisie montante, sa préoccupation pour les questions de préséance et d'étiquette à la cour, son regard perçant pour reconnaître les traits affectés particuliers à chacun, (...) » Cité par Luc Fraisse, art. cit., p. 64.

64 Proust, op. cit., éd. Jean Milly, p. 266 sqq.

65 Ibid. p. 279.

66 Trésor de la langue française (informatisé).

67 Jean Milly, op. cit., p. 32.

68 Gérard Genette, Figures I, op. cit., p. 44.

69 Florence Mercier-Leca, art. cit., p. 112.

70 Proust, op. cit., éd. Jean Milly, p. 238.

71 Proust, op. cit., éd. Jean Milly, p. 228.

72 Ibid. p. 24.

73 Ibid. p. 270.

74 Ibid. p. 271.

75 Voir. Yves Sandre, «Proust Chroniqueur », Revue d'histoire littéraire de la France, n. 5-6, 9-12/1971, p.776.

76 Gérard Genette, Figures III, op. cit., p. 39. 
77 Proust, op. cit., éd. Jean Milly, p. 274.

$78 \quad$ Ibid. p. 308.

79 Florence Mercier-Leca, art. cit., p. 204.

80 Ibid.

81 Ibid.

82 Ibid.

83 Proust, op. cit., éd. Jean Milly, p.257.

84 Roland Barhtes, op. cit., p. 7.

85 Paul Aron, «Sur les pastiches de Proust l'ethos et le champ », art. cit., p. 10.

86 Proust, op. cit., éd. Jean Milly, p. 231.

87 Ibid. p. 295.

88 Ibid. p. 258.

89 Ibid. p. 296.

$90 \quad$ Ibid. p. 305.

91 Ibid. p. 316.

92 Ibid. p. 317.

93 Ibid. p. 268.

94 Ibid. p. 271.

95 Ibid. p. 272.

96 Ibid. p. 308.

97 Ibid. p. 311.

$98 \quad$ Ibid. p. 312.

99 Ibid. p. 283.

100 Ibid. p. 315.

101 Ibid.

102 Ibid. p. 317.

103 Saint-Simon, op. cit., éd. Yves Coirault, p. 504.

104 Roland Barthes, op. cit., p. 24.

105 Proust, op. cit., éd. Jean Milly, p. 270.

106 Ibid. p. 289.

107 Robert Montesquiou, Les Perles Rouges, Charpentier et Fasquelle, 1899, p. 26.

108 Gérard Genette, Figures III, op. cit., p. 72.

109 Luc Fraisse, art. cit., p.70.

110 Gérard Genette, Figures III, op. cit., p. 184.

111 Gérard Genette, Palimpsestes, op. cit., p. 114.

112 Roland Barthes, Le Degré Zéro de l'écriture. Éditions du Seuil, 1953, p. 60.

113 Proust, op. cit., éd. Jean Milly, p. 278.

114 Gérard Genette, Figures III, op. cit., p. 187.

115 Ibid. p. 263.

116 Ibid. p. 226.

117 Ibid. p. 259.

118 Jean-Michel Delacomptée, La Grandeur, Saint-Simon, Gallimard, coll. L'un et l'autre, 2011, p. 19 sqq.

119 Proust, op. cit., éd. Jean Milly, p. 241.

120 Luc Fraisse, art. cit., p. 65.

121 Gérard Genette, Figures I, op. cit., p. 167.

122 Proust, op. cit., éd. Jean Milly, p. 229 ssq.

123 Ibid. p. 303.

124 Ibid. p. 267. 
125 Ibid. p. 233.

126 Ibid. p. 226.

127 Robert Montesquiou, Les Perles Rouges, Les Paroles Diaprées, 1910, p. 45.

128 Proust, op. cit., éd. Jean Milly, p. 226.

129 Ibid.

\section{Bibliographie}

Paul Aron, "Les pastiches littéraires dans À la recherche du temps perdu ", Revue d'histoire littéraire de la France, 2012/1 Vol. 112, p. 51-61.

Paul Aron, "Sur les pastiches de Proust l'ethos et le champ " sur le site revues. org/contextes.

Roland Barthes. Introduction à l'analyse structurale des récits. In: Communications, 8/1966. Recherches sémiologiques : l'analyse structurale du récit.

Roland Barthes, Le Degré Zéro de l'écriture. Éditions du Seuil, 1953.

D. C. Cabeen, "Proust and Saint-Simon ", Modern Language Association, Vol. 46, No. 2, 6/1931, p. 608-618.

Jean-Michel Delacomptée, La Grandeur, Saint-Simon, Gallimard, coll. L'un et l'autre, 2011.

Denis Delphine, " «À la manière de» : le pastiche avant le pastiche ", Revue d'histoire littéraire de la France, 2012/1 Vol. 112, p. 7-18.

Luc Fraisse, "Proust : philosophie du pastiche et pastiche de la philosophie ", Revue d'histoire littéraire de la France, 2012/1 Vol. 112, p. 63-75.

Gérard Genette, Figures I, Éditions du Seuil, coll. Points, 1966.

Gérard Genette, Figures III, Éditions du Seuil, coll. Poétique, 1972.

Gérard Genette, Palimpsestes, Éditions du Seuil, coll. Points, 1982.

Bille Jørgensen Steen, « Pastiche et poétique de l'œuvre. Stratégies de réécriture contemporaines ", Revue d'histoire littéraire de la France, 2012/1 Vol. 112, p.105-117.

Florence Mercier-Leca, "Saint-Simon au miroir : trois pastiches des Mémoires aux XXe et XXIe siècles », Littératures classiques, 2011/1 N 74, p. 201-217.

Robert Montesquiou, Les Perles Rouges, Les Paroles Diaprées, 1910.

Marcel Proust, Les Pastiches de Proust, édition critique et commentée par Jean Milly, Armand Colin, 1970.

Saint-Simon, Mémoires, éd. Yves Coirault, Gallimard, coll. Folio, 1990.

Yves Sandre, "Proust Chroniqueur ", Revue d'histoire littéraire de la France, n.5-6, 9-12/1971, p. 771-790. 
